\title{
An Isomerisation Approach to Tesirine and Pyrrolobenzodiazepines
}

\author{
Andrew D. Campbell ${ }^{\dagger}$, Simone Tomasi ${ }^{\dagger}$, Arnaud C. Tiberghien ${ }^{\ddagger}$, and Jeremy S. Parker ${ }^{\S *}$ \\ ${ }^{\dagger}$ Chemical Development, Pharmaceutical Technology \& Development, AstraZeneca, Macclesfield, SK10 2NA, \\ United Kingdom \\ \$Spirogen, QMB Innovation Centre, 42 New Road, E1 2AX London, United Kingdom \\ ${ }^{\S}$ Early Chemical Development, Pharmaceutical Sciences, R\&D, AstraZeneca, Macclesfield, United Kingdom \\ jeremy.parker@astrazeneca.com
}

\section{Contents}

S2 Large scale conversion of 5-methoxy-2-nitro-4-((triisopropylsilyl)oxy)benzoic acid (4) to (S)(2-(((tert-butyldimethylsilyl)oxy)methyl)-4-methylenepyrrolidin-1-yl)(5-methoxy-2-nitro-4((triisopropylsilyl)oxy)phenyl)methanone (8)

S11 Catalyst Screening

S26 Reaction Profiling

S28 4-Methyl-2,5-dihydropyrrole (11) and 2-Methylene-4-methylpyrrolidine (12) Isomers

S36 Computational Estimates for the Thermodynamics of the Isomerisation Reactions

S38 Chiral analysis of 4-Methyl-2,3-dihydropyrrole (6)

S40 NMR spectra of 6 prepared using isomerisation 
Large scale conversion of 5-methoxy-2-nitro-4-((triisopropylsilyl)oxy)benzoic acid (4) to $(S)$-(2(((tert-butyldimethylsilyl)oxy)methyl)-4-methylenepyrrolidin-1-yl)(5-methoxy-2-nitro-4((triisopropylsilyl)oxy)phenyl)methanone (8)

Preparation of methyl (S)-1-(5-methoxy-2-nitro-4-((triisopropylsilyl)oxy)benzoyl)-4methylenepyrrolidine-2-carboxylate<smiles>COc1cc(C(=O)O)c([N+](=O)[O-])cc1OC(F)(F)F</smiles>

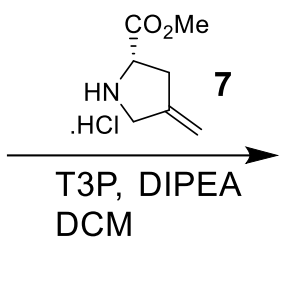<smiles>C=C1C[C@H](C(C)=O)N(C(=O)c2cc(OC)c(OS(C)(=O)=O)cc2[N+](=O)[O-])C1</smiles>

Propanephosphonic acid anhydride (T3P, 1.91kg, 6.00mol, 2.0eq), followed by DIPEA (3.14L, 18.0mol, 6.0eq) were added dropwise at $-15^{\circ} \mathrm{C}$ to a mixture of 5-methoxy-2-nitro-4((triisopropylsilyl)oxy)benzoic acid $(4,1.11 \mathrm{~kg}, 3.00 \mathrm{~mol}, 1.0 \mathrm{eq})$ and methyl $(S)$-4-methylenepyrrolidine2-carboxylate hydrochloride $(7,640 \mathrm{~g}, 3.60 \mathrm{~mol}, 1.2 \mathrm{eq})$ in dichloromethane (12L, 10.8vol). The reaction mixture was allowed to stir at $-15^{\circ} \mathrm{C}$ for $1 \mathrm{~h}$. Reaction completion was observed by HPLC. The reaction was quenched with water $(12 \mathrm{~L}, 10.8 \mathrm{vol})$ at $5{ }^{\circ} \mathrm{C}$. The organic phase was washed with $10 \%(\mathrm{w} / \mathrm{w})$ aqueous citric acid (12L, 10.8vol), brine (12L, 10.8vol), and dried over sodium sulfate. The volatiles were removed under reduced pressure and the residue was purified by chromatography (heptane/ethyl acetate gradient from $90 / 10$ to $75 / 25)$ to give methyl (S)-1-(5-methoxy-2-nitro-4((triisopropylsilyl)oxy)benzoyl)-4-methylenepyrrolidine-2-carboxylate $(1.1 \mathrm{~kg}, 74 \%$, LC purity $99.8 \%)$ as light yellow oil.

${ }^{1} \mathrm{H}$ NMR (400 MHz, $\mathrm{CDCl}_{3}$, two atropisomers in a 1/1.74 ratio) $7.75-7.62(\mathrm{~m}, 1 \mathrm{H}), 6.89-6.74(\mathrm{~m}$, $1 \mathrm{H}), 5.19-4.54(\mathrm{~m}, 3 \mathrm{H}), 4.29-3.50(\mathrm{~m}, 8 \mathrm{H}), 3.15-2.95(\mathrm{~m}, 1 \mathrm{H}), 2.79-2.57(\mathrm{~m}, 1 \mathrm{H}), 1.37-1.19$ $(\mathrm{m}, 3 \mathrm{H}), 1.17-1.01(\mathrm{~m}, 18 \mathrm{H})$.

${ }^{13} \mathrm{C}$ NMR (101 MHz, $\left.\mathrm{CDCl}_{3}\right)$ 172.10, 171.97, 166.90, 166.67, 156.62, 156.21, 146.19, 146.14, 142.01, 141.54, 137.38, 127.34, 126.88, 116.21, 116.11, 110.67, 109.93, 109.16, 109.06, 60.76, 58.32, 56.33, $56.21,52.63,52.51,52.22,50.44,37.32,35.71,17.93,17.90,12.93,12.90$.

IR $\left(\mathrm{cm}^{-1}\right): 2946,2868,1745,1652,1570,1522,1421,1334,1290,1223,1063,884,841,684$.

$[\alpha]^{21}{ }_{D}=-12^{\circ}$.

HRMS (ESI) m/z: Calcd for $\mathrm{C}_{24} \mathrm{H}_{36} \mathrm{~N}_{2} \mathrm{O}_{7} \mathrm{Si}$ 493.23645; Measured 493.23657. 
Preparation of (S)-(2-(hydroxymethyl)-4-methylenepyrrolidin-1-yl)(5-methoxy-2-nitro-4((triisopropylsilyl)oxy)phenyl)methanone<smiles>C=C1C[C@H](C(C)=O)N(C(=O)c2cc(OC)c(OS(C)(=O)=O)cc2[N+](=O)[O-])C1</smiles><smiles>CO[C@H](C)C(C)C</smiles><smiles>C=C1C[C@H](CO)N(C(=O)c2cc(OC)c(OC(F)(F)F)cc2[N+](=O)[O-])C1</smiles>

Sodium borohydride $(180 \mathrm{~g}, 4.75 \mathrm{~mol}, 2.5 \mathrm{eq})$, was added at $0^{\circ} \mathrm{C}$ to a solution of $(S)-1$-(5-methoxy-2nitro-4-((triisopropylsilyl)oxy)benzoyl)-4-methylenepyrrolidine-2-carboxylate $(935 \mathrm{~g}, 1.90 \mathrm{~mol}, 1.0 \mathrm{eq})$ in tetrahydrofuran $(5.6 \mathrm{~L}, 6 \mathrm{vol})$. Methanol $(1.9 \mathrm{~L}, 2 \mathrm{vol})$ was then added at $0^{\circ} \mathrm{C}$ and the reaction mixture was allowed to stir at $0^{\circ} \mathrm{C}$ for $2 \mathrm{~h}$. Reaction completion was observed by HPLC. The reaction was diluted with cold DCM $(6.5 \mathrm{~L}, 7 \mathrm{vol})$ and quenched with $10 \%(\mathrm{w} / \mathrm{w})$ aqueous citric acid $(6.2 \mathrm{~L}, 6.6 \mathrm{vol})$ at $5^{\circ} \mathrm{C}$, followed by water $(5.6 \mathrm{~L}, 6 \mathrm{vol})$. The aqueous layer was extracted with DCM $2 \times(5.6 \mathrm{~L}, 6 \mathrm{vol})$. The organics were washed with saturated aqueous sodium bicarbonate $(5.6 \mathrm{~L}, 6 \mathrm{vol})$, brine $(5.6 \mathrm{~L}, 6 \mathrm{vol})$, and dried over sodium sulfate. The volatiles were removed under reduced pressure and the residue was purified by chromatography (heptane/ethyl acetate gradient from 80/20 to 50/50) to give $(S)$-(2(hydroxymethyl)-4-methylenepyrrolidin-1-yl)(5-methoxy-2-nitro-4-((triisopropylsilyl)oxy)phenyl) methanone $(0.83 \mathrm{~kg}, 94 \%$, LC purity $97.5 \%)$ as light yellow oil.

${ }^{1} \mathrm{H}$ NMR (400 MHz, $\mathrm{CDCl}_{3}$, Atropisomers in 0.14/1 ratio) $7.76-7.61(\mathrm{~m}, 1 \mathrm{H}), 6.94-6.69(\mathrm{~m}, 1 \mathrm{H})$, $5.05-4.80(\mathrm{~m}, 2 \mathrm{H}), 4.68-4.53(\mathrm{~m}, 1 \mathrm{H}), 3.98-3.48(\mathrm{~m}, 8 \mathrm{H}), 2.94-2.75(\mathrm{~m}, 1 \mathrm{H}), 2.60-2.40(\mathrm{~m}, 1 \mathrm{H})$, $1.37-1.19(\mathrm{~m}, 3 \mathrm{H}), 1.17-1.01(\mathrm{~m}, 18 \mathrm{H})$.

${ }^{13} \mathrm{C}$ NMR $\left(101 \mathrm{MHz}, \mathrm{CDCl}_{3}\right) 156.79,146.19,142.79,137.26,127.65,116.20,109.50,108.36,60.47$, $56.35,53.08,34.50,17.91,12.92$.

IR $\left(\mathrm{cm}^{-1}\right)$ 2945, 2867, 1622, 1570, 1522, 1431, 1334, 1291, 1225, 1060, 884, 842, 684.

$[\alpha]^{21}=-34^{\circ}$.

HRMS (ESI) m/z: Calcd for $\mathrm{C}_{23} \mathrm{H}_{36} \mathrm{~N}_{2} \mathrm{O}_{6} \mathrm{Si}$ 465.24154; Measured 465.24176 


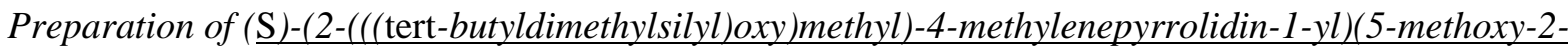
nitro-4-((triisopropylsilyl)oxy)phenyl)methanone (8)<smiles>C=C1C[C@H](CO)N(C(=O)c2cc(OC)c(OC(F)(F)F)cc2[N+](=O)[O-])C1</smiles>

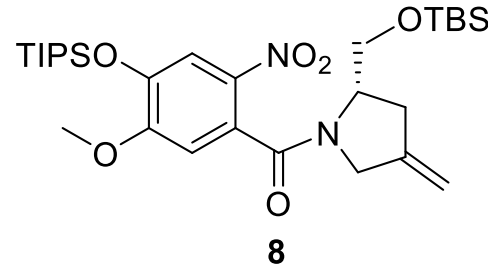

(S)-(2-(hydroxymethyl)-4-methylenepyrrolidin-1-yl)(5-methoxy-2-nitro-4-((triisopropylsilyl)oxy) phenyl)methanone $(837 \mathrm{~g}, 1.79 \mathrm{~mol})$ and imidazole $(244 \mathrm{~g}, 3.59 \mathrm{~mol}, 2.0 \mathrm{eq})$ were dissolved in dichloromethane $(5.0 \mathrm{~L}, 6 \mathrm{vol})$ at room temperature. $\mathrm{TBSCl}(404 \mathrm{~g}, 2.68 \mathrm{~mol}, 1.5 \mathrm{eq})$ was added portionwise whilst keeping the temperature below $30^{\circ} \mathrm{C}$. The reaction mixture was stirred at $25^{\circ} \mathrm{C}$ for 1h. Reaction completion was observed by HPLC. The solids were removed by filtration and washed with dichloromethane $(1.7 \mathrm{~L}, 2 \mathrm{vol})$. The solution was washed with water $(4.2 \mathrm{~L}, 5 \mathrm{vol})$, then brine $(4.2 \mathrm{~L}$, $5 \mathrm{vol})$, and dried over sodium sulfate. The volatiles were removed under vacuum and the residue was purified by filtration through a short pad of silica gel (heptane/ethyl acetate) to give $(S)-(2-(((t e r t-$ butyldimethylsilyl)oxy)methyl)-4-methylenepyrrolidin-1-yl)(5-methoxy-2-nitro-4-

((triisopropylsilyl)oxy)phenyl)methanone $(\mathbf{8}, 1010 \mathrm{~g}, 1.74 \mathrm{~mol}, 97 \%$ yield, LC purity $99 \%)$ as a yellow oil.

${ }^{1} \mathrm{H}$ NMR (400 MHz, $\mathrm{CDCl}_{3}$, Atropisomers in 2/1 ratio) $7.78-7.57(\mathrm{~m}, 1 \mathrm{H}), 6.84-6.64(\mathrm{~m}, 1 \mathrm{H}), 5.19$ $-4.45(\mathrm{~m}, 3 \mathrm{H}), 4.07-3.64(\mathrm{~m}, 6 \mathrm{H}), 3.62-3.18(\mathrm{~m}, 1 \mathrm{H}), 2.90-2.41(\mathrm{~m}, 2 \mathrm{H}), 1.38-1.18(\mathrm{~m}, 3 \mathrm{H}), 1.15$ $-1.03(\mathrm{~m}, 18 \mathrm{H}), 0.96-0.72(\mathrm{~m}, 9 \mathrm{H}), 0.15--0.20(\mathrm{~m}, 6 \mathrm{H})$.

${ }^{13} \mathrm{C}$ NMR (101 MHz, $\left.\mathrm{CDCl}_{3}\right)$ 166.69, 166.43, 156.40, 156.12, 145.99, 145.78, 144.20, 143.36, 137.39, 137.31, 128.07, 127.25, 116.04, 110.42, 109.51, 108.28, 107.42, 63.73, 62.58, 60.21, 58.14, 56.09, $56.07,52.76,50.57,35.00,33.97,25.85,25.76,25.67,18.20,17.99,17.81,17.72,17.65,13.10,12.82$, $12.80,12.51,-3.57,-5.41,-5.43,-5.58$. 


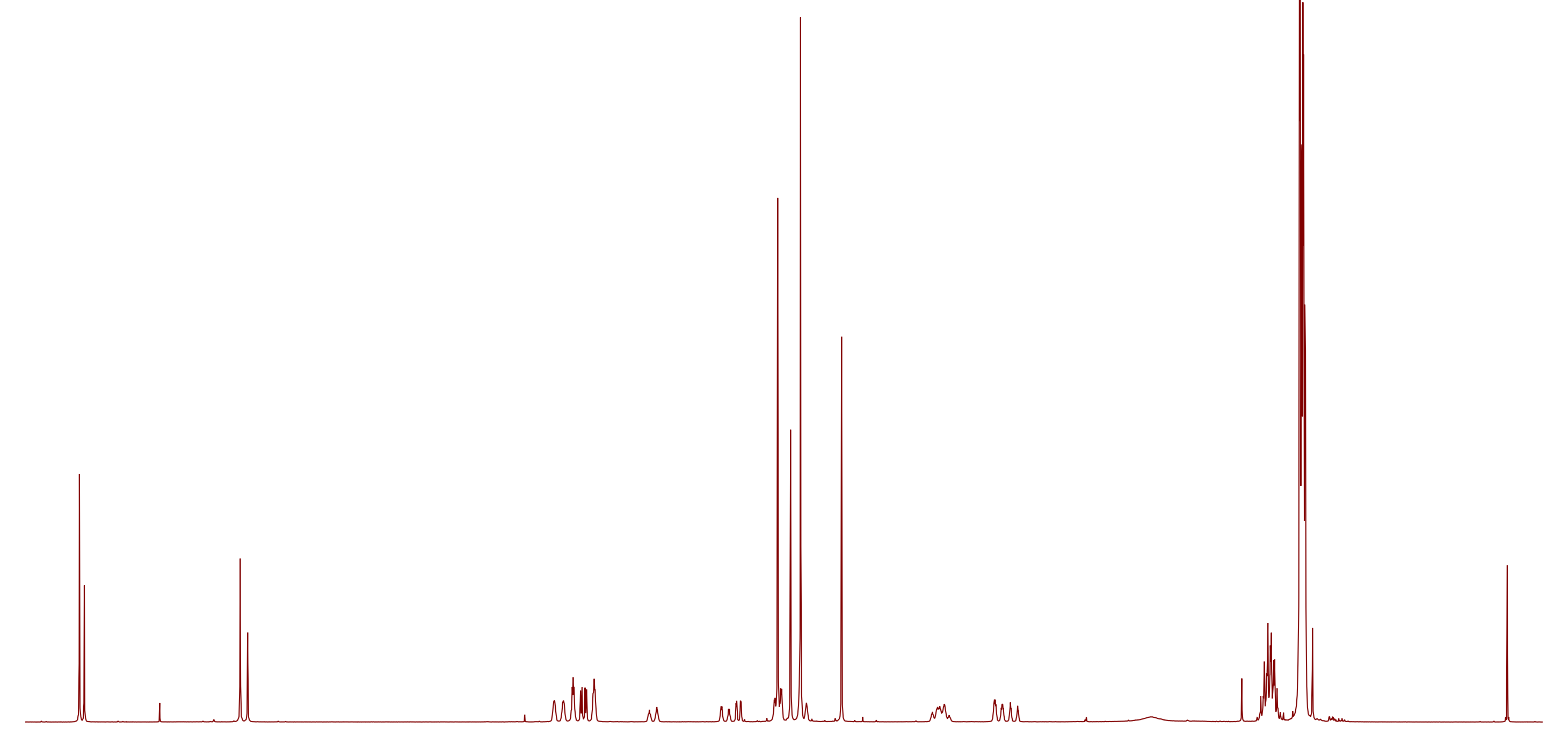

$\begin{array}{llllllllllllllll}7.5 & 7.0 & 6.5 & 6.0 & 5.5 & 5.0 & 4.5 & \begin{array}{c}4.0 \\ \mathrm{f} 1(\mathrm{ppm})\end{array} & 3.5 & 3.0 & 2.5 & 2.0 & 1.5 & 1.0 & 0.5 & 0.0\end{array}$




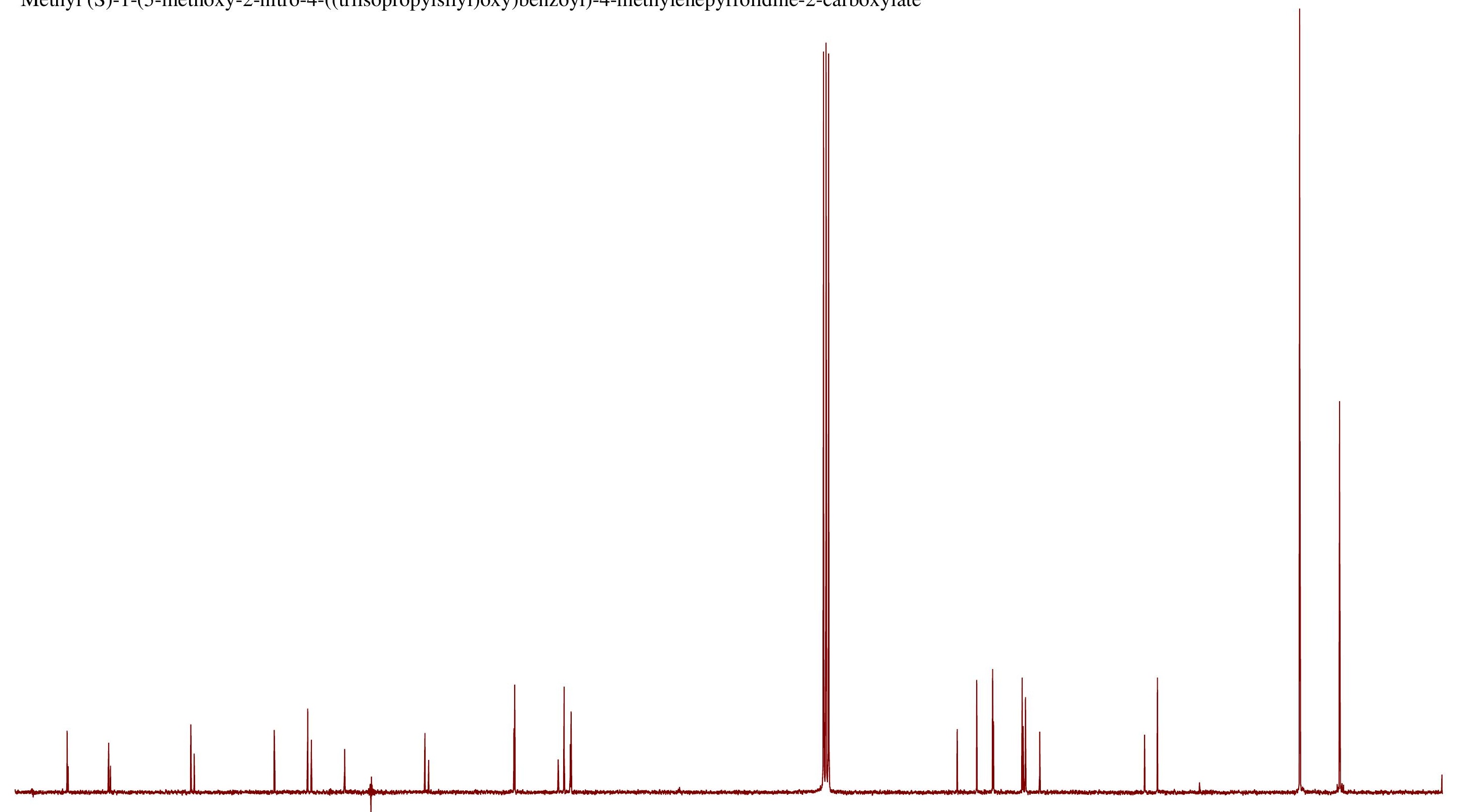

$\begin{array}{llllllllllllllllll}170 & 160 & 150 & 140 & 130 & 120 & 110 & 100 \underset{\mathrm{f} 1(\mathrm{ppm})}{90} 80 & 70 & 60 & 50 & 40 & 30 & 20 & 10 & c\end{array}$




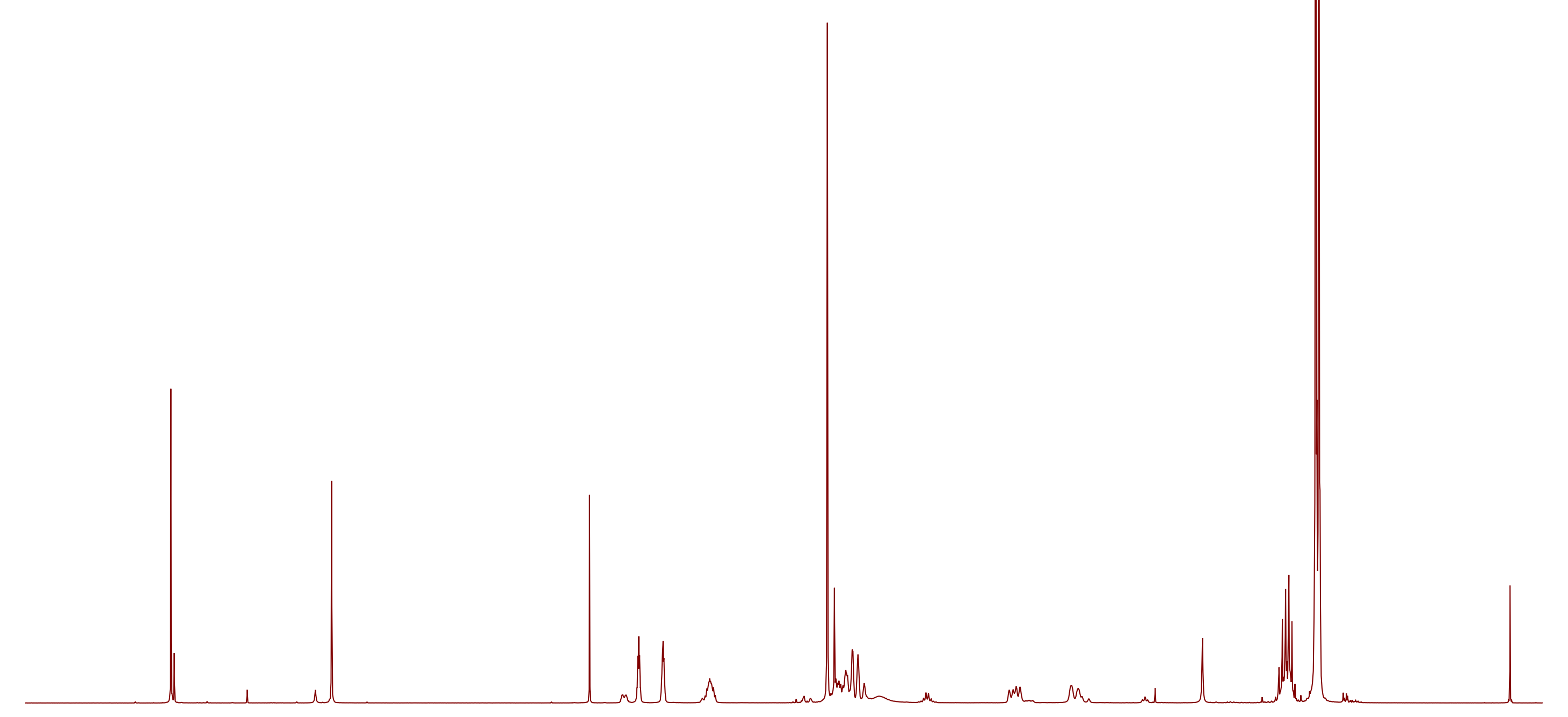

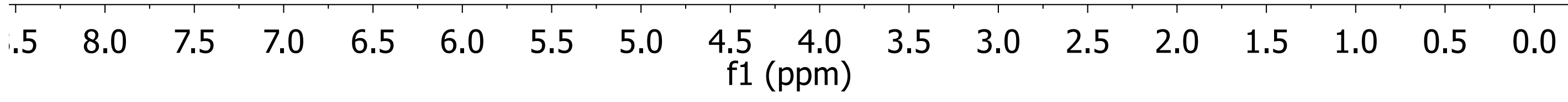




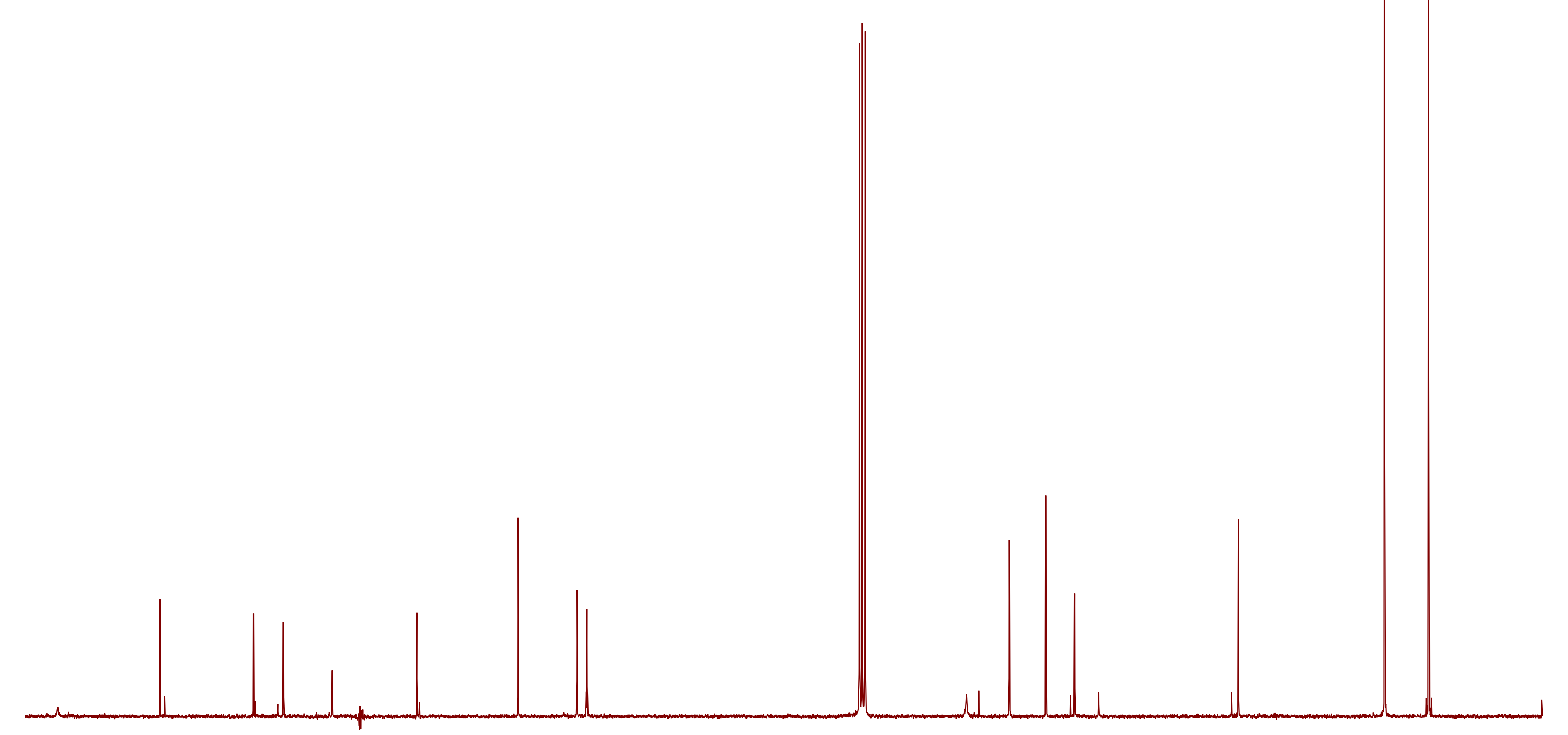

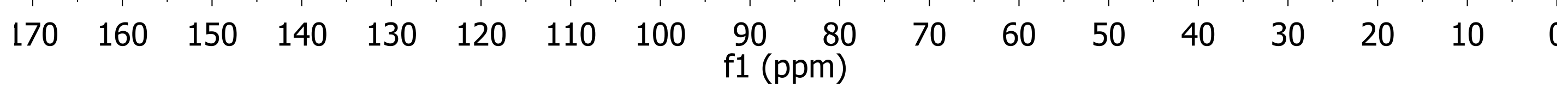


(S)-(2-(((tert-butyldimethylsilyl)oxy)methyl)-4-methylenepyrrolidin-1-yl)

(5-methoxy-2-nitro-4-((triisopropylsilyl)oxy)phenyl)methanone (8)

$\begin{array}{llllllllllllllllll}8.0 & 7.5 & 7.0 & 6.5 & 6.0 & 5.5 & 5.0 & 4.5 & \begin{array}{c}4.0 \\ \mathrm{f} 1(\mathrm{ppm})\end{array} & 3.5 & 2.5 & 2.0 & 1.5 & 1.0 & 0.5 & 0.0 & -0.5\end{array}$




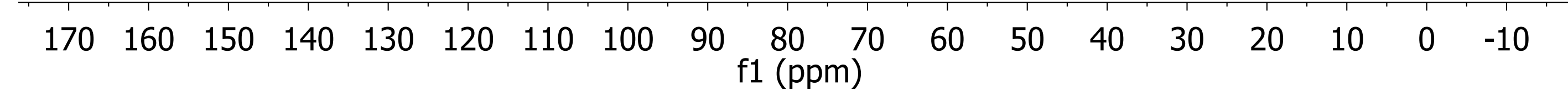




\section{Catalyst Screening}

Screening work completed on conversion of (S)-(2-((tert-butyldimethylsilyloxy) methyl)-4methylenepyrrolidin-1-yl)(5-methoxy-2-nitro-4-(triisopropylsilyloxy) phenyl)methanone $(8)$ to $(S)-(2-$ ((tert-butyldimethylsilyloxy)methyl)-4-methyl-2,3-dihydro-1H-pyrrol-1-yl)(5-methoxy-2-nitro-4(triisopropylsilyloxy)phenyl)methanone (6)

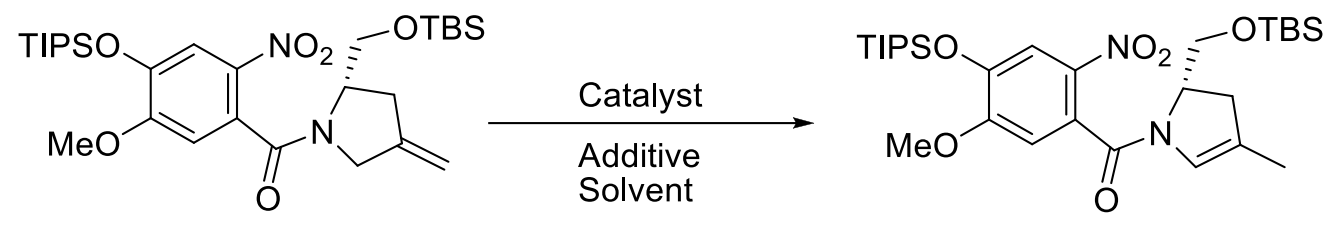

\section{$\underline{\text { Screen } 1}$}

Initial isomerisation screen to asses a wide range of methods

Catalyst selection made by literature review and in-house knowledge.

\section{Experimental Set-Up}

- On a $100 \mathrm{mg}$ scale of alkene, reactions were performed in $4 \mathrm{~mL}$ vials in a 24 -well plate format, situated in an inerted glovebox $\left(<10 \mathrm{ppm}_{2}\right.$ and $\left.<1 \mathrm{ppm} \mathrm{H}_{2} \mathrm{O}\right)$

- Metal sources were weighed by hand (if air insensitive) or were dispensed as solids using a Quantos weighing robot situated inside the glovebox

- Stir discs were added to each vial

- Starting material $(2 \mathrm{~g})$ was dissolved in dry degassed toluene $(10 \mathrm{~mL}$ total $)$ and $500 \mu \mathrm{L}$ was dispensed to each vial (100mg/reaction)

- Each vial was then made up to $2 \mathrm{~mL}$ with the solvent stated in the reaction plan

- Liquid additives (reactions 2, 5 and 16) were then added

- The reactions were sealed and heated at $60^{\circ} \mathrm{C}$ for $4 \mathrm{~h}$ then at $100^{\circ} \mathrm{C}$ for a further $18 \mathrm{~h}$ for all reactions except reaction 4 (in $\mathrm{MeOH}$, kept at $60^{\circ} \mathrm{C}$ )

- Samples for UPLC/MS analysis were prepared at $1 \mathrm{~h}$ and $22 \mathrm{~h}$.

UPLC Analysis:

Column: Phenomenex Kinetex 2.6u C18 100A 75mm x 3mm

Mobile phase A: $0.03 \%$ TFA in water

Mobile phase B: $0.025 \%$ TFA in acetonitrile

Gradient:

\begin{tabular}{|l|l|l|}
\hline Time & $\% \mathrm{~A}$ & $\% \mathrm{~B}$ \\
\hline 0.00 & 30 & 70 \\
\hline 8.00 & 15 & 85 \\
\hline 8.50 & 5 & 95 \\
\hline
\end{tabular}

Flow rate: $1.2 \mathrm{~mL} / \mathrm{min}$

Detection: UV @ 220nm

Temperature: $40^{\circ} \mathrm{C}$ 
Reaction Plan and Results

\begin{tabular}{|c|c|c|c|c|c|c|c|c|c|c|c|c|c|c|c|}
\hline \multirow[b]{2}{*}{$\begin{array}{l}\text { Reaction } \\
\text { Number }\end{array}$} & \multirow[b]{2}{*}{ Catalyst } & \multirow[b]{2}{*}{$\begin{array}{l}\text { Catalyst } \\
\text { (mol\%) }\end{array}$} & \multirow[b]{2}{*}{ Additive } & \multirow[b]{2}{*}{$\begin{array}{c}\text { Additive } \\
\text { eq. }\end{array}$} & \multirow[b]{2}{*}{$\begin{array}{l}\text { Alkene } \\
\text { (SM) }\end{array}$} & \multirow[b]{2}{*}{$\begin{array}{c}\text { Temp. } \\
{ }^{\circ} \mathrm{C}\end{array}$} & \multirow[b]{2}{*}{$\begin{array}{l}\text { Solvent } \\
\text { (20vols) }\end{array}$} & \multirow[b]{2}{*}{$\begin{array}{c}\text { Conversion } \\
\text { at } 1 \mathrm{~h}\end{array}$} & \multirow[b]{2}{*}{$\begin{array}{c}\text { Product/ } \\
\text { Isomer } \\
\text { ratio } \\
\end{array}$} & \multirow[b]{2}{*}{$\begin{array}{c}\text { Conversion } \\
\text { at } 22 \mathrm{~h}\end{array}$} & \multirow[b]{2}{*}{$\begin{array}{c}\text { Product/ } \\
\text { Isomer } \\
\text { ratio } \\
\end{array}$} & \multicolumn{4}{|c|}{ Area\% at $220 \mathrm{nM}$ at $22 \mathrm{~h}$} \\
\hline & & & & & & & & & & & & Product & $\begin{array}{c}\text { isomeric } \\
\text { product }\end{array}$ & SM & $\begin{array}{l}\text { Other } \\
\text { peaks }\end{array}$ \\
\hline 1 & Grubbs I & 5 & none & & 1.0 eq. & $60-100$ & Toluene & 0.0 & 0.0 & 20.6 & 0.6 & 7.7 & 12.4 & 77.9 & 2.0 \\
\hline 2 & Grubbs I & 5 & Et3SiH & 1 & 1.0 eq. & $60-100$ & Toluene & 0.0 & 0.0 & 32.1 & 1.1 & 12.3 & 11.0 & 49.1 & 27.7 \\
\hline 3 & Grubbs II & 5 & none & & 1.0 eq. & $60-100$ & Toluene & 0.0 & 0.0 & 19.5 & 5.3 & 14.5 & 2.7 & 71.1 & 11.7 \\
\hline 4 & Grubbs II & 5 & none & & $1.0 \mathrm{eq}$. & 60 & $\mathrm{MeOH}$ & 19.5 & 1.2 & 100.0 & 0.9 & 46.2 & 52.4 & 0.0 & 1.4 \\
\hline 5 & none & & $\mathrm{Fe}(\mathrm{CO}) 5$ & 3 & $1.0 \mathrm{eq}$. & $60-100$ & CPME & 0.0 & 0.0 & 0.0 & 0.0 & 0.0 & 0.0 & 70.1 & 29.9 \\
\hline 6 & Crabtrees Cat. & 5 & none & & 1.0 eq. & $60-100$ & Toluene & 0.0 & 0.0 & 13.8 & 22.1 & 12.1 & 0.5 & 78.8 & 8.6 \\
\hline 7 & Crabtrees Cat. & 5 & none & & $1.0 \mathrm{eq}$. & $60-100$ & IPA/Toluene & 0.0 & 0.0 & 100.0 & 0.8 & 29.2 & 36.9 & 0.0 & 33.9 \\
\hline 8 & $\mathrm{Ru}(\mathrm{H} 2)(\mathrm{PPh} 3) 4$ & 5 & none & & 1.0 eq. & $60-100$ & Toluene & 0.0 & 0.0 & 0.0 & 0.0 & 0.0 & 0.0 & 95.2 & 4.8 \\
\hline 9 & $\mathrm{RuHCl}(\mathrm{CO}) \mathrm{PPh} 3$ & 5 & none & & $1.0 \mathrm{eq}$. & $60-100$ & Toluene & 18.5 & 0.1 & 100.0 & 0.4 & 26.4 & 68.6 & 0.0 & 5.0 \\
\hline 10 & cationic $\mathrm{CpRu}(\mathrm{Pr} 3)$ (strem) & 5 & none & & 1.0 eq. & $60-100$ & Toluene & 83.5 & 0.3 & 100.0 & 0.4 & 25.2 & 71.4 & 0.0 & 3.3 \\
\hline 11 & $\mathrm{RhH}(\mathrm{CO}) \mathrm{PPh} 3$ & 5 & none & & $1.0 \mathrm{eq}$. & $60-100$ & Toluene & 21.2 & 0.0 & 47.2 & 0.2 & 6.3 & 38.6 & 50.3 & 4.8 \\
\hline 12 & $\mathrm{RhCl} 3 . \mathrm{H} 20$ & 5 & none & & $1.0 \mathrm{eq}$. & $60-100$ & $\mathrm{nBuOH}$ & 0.0 & 0.0 & 0.0 & 0.0 & 0.0 & 0.0 & 0.0 & 100.0 \\
\hline 13 & $\mathrm{Rh}(\mathrm{COD}) 2 \mathrm{BF} 4$ & 5 & Binap & 0.05 & $1.0 \mathrm{eq}$. & $60-100$ & Toluene & 0.0 & 0.0 & 100.0 & 0.4 & 25.1 & 56.6 & 0.0 & 18.3 \\
\hline 14 & $\mathrm{Pd} / \mathrm{C}$ & 5 & none & & $1.0 \mathrm{eq}$. & $60-100$ & Toluene & 0.0 & 0.0 & 0.0 & 0.0 & 0.0 & 0.0 & 99.7 & 0.3 \\
\hline 15 & $\mathrm{Pd}-113$ & 5 & none & & $1.0 \mathrm{eq}$. & $60-100$ & Toluene & 100.0 & 2.4 & 100.0 & 15.7 & 89.5 & 5.7 & 0.0 & 4.8 \\
\hline 16 & $\mathrm{Pd}-118$ & 5 & Et3SiH & 0.1 & $1.0 \mathrm{eq}$. & $60-100$ & Toluene & 97.0 & 0.3 & 100 & 0.3 & 20.5 & 64.4 & 0.0 & 15.2 \\
\hline 17 & $\mathrm{Pd}(\mathrm{MeCN}) 2 \mathrm{Cl} 2$ & 5 & none & & $1.0 \mathrm{eq}$. & $60-100$ & Toluene & 0.0 & 0.0 & 0 & 0 & 0.0 & 0.0 & 91.4 & 8.6 \\
\hline 18 & $\mathrm{Pd}(\mathrm{OAc}) 2 / \mathrm{PhS}(\mathrm{O})(\mathrm{CH} 2) 2 \mathrm{~S}(\mathrm{O}) \mathrm{Ph}$ & 5 & none & & $1.0 \mathrm{eq}$. & $60-100$ & Toluene & 0.0 & 0.0 & 0 & 0 & 0.0 & 0.0 & 93.5 & 6.5 \\
\hline
\end{tabular}

$\operatorname{Conversion}(\%)=\frac{100 *(\text { Product Area } \%+\text { Isomeric product Area } \%)}{(\text { SM Area } \%)+(\text { Product Area } \%)+(\text { Isomeric product Area } \%)}$ 
Reaction Products and Impurities

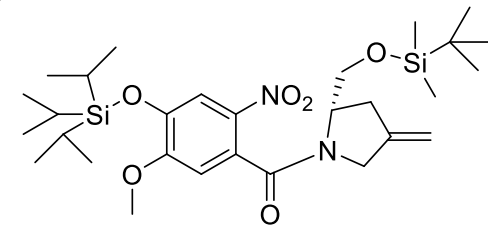

Starting material, $\mathrm{Rt}=7.9 \mathrm{mins}$ Molecular Weight: 578.89

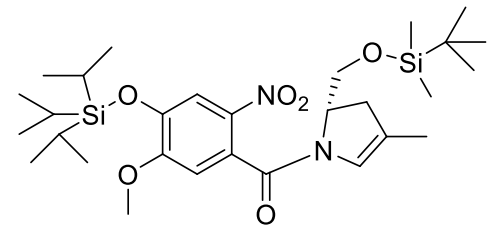

Product, $\mathrm{Rt}=8.2 \mathrm{mins}$ Molecular Weight: 578.89

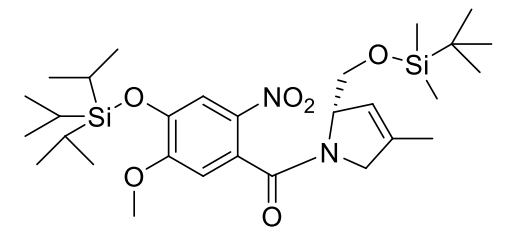

Isomeric product, $\mathbf{R t}=7.6 \mathrm{mins}$ Molecular Weight: 578.89

Typical HPLCs (toluene peak at $0.5 \mathrm{mins}$ )

(Reaction 15, Pd-113 (5mol\%), toluene, 22h)

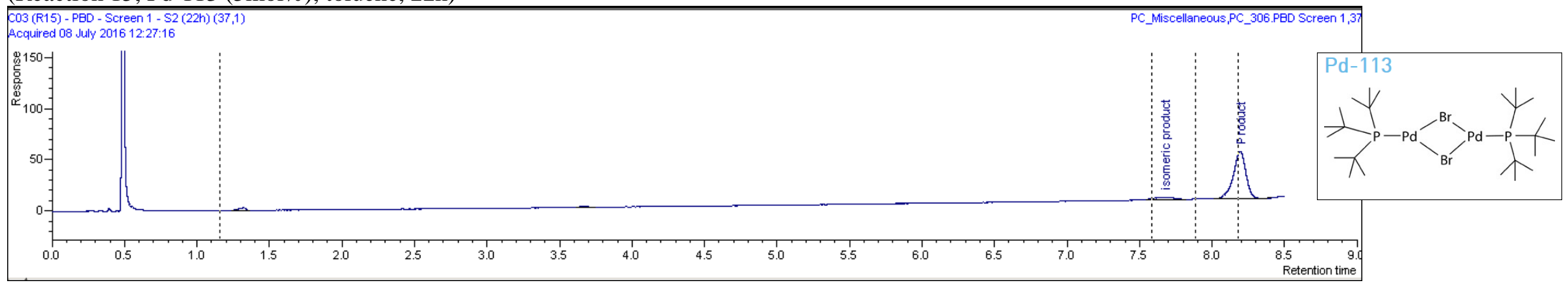

(Reaction 10, Cationic Ru catalyst ( $5 \mathrm{~mol} \%$ ), toluene, $22 \mathrm{~h}$ )

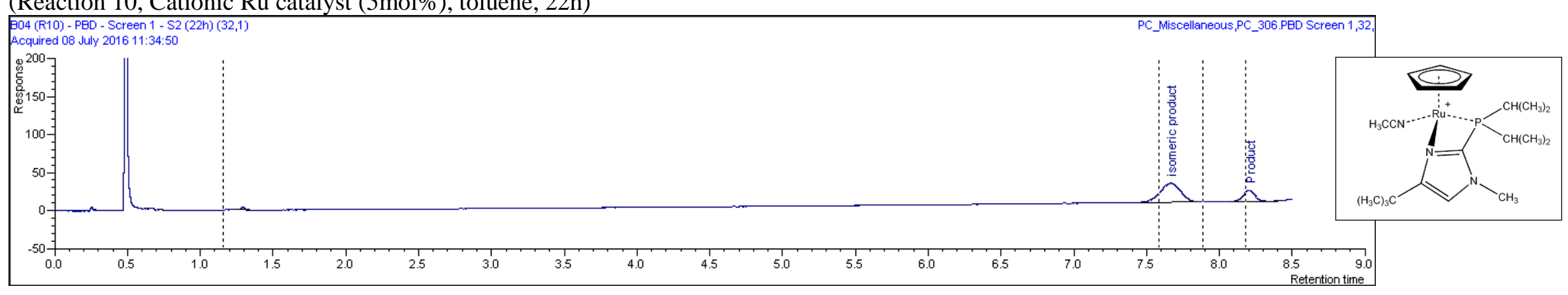




\section{Conclusions}

- Product is formed in a number of reactions

- The best reaction uses Pd-113 which provides relatively clean product ( $89 \%$ by HPLC) with small amounts of isomeric product

- For Pd-113, the isomeric product appears to be an intermediate which progresses on to the desired product (data from $1 \mathrm{~h}$ and $22 \mathrm{~h}$ HPLC samples)

- $5 \mathrm{~mol} \%$ of $\mathrm{Pd}-113$ is actually $10 \mathrm{~mol} \%$ of $\mathrm{Pd}$ as the structure is dimeric

- A Pd-mirror is formed in this reaction

- A number of metal hydride type catalysts do give product although the selectivity for product appears to be poor

$\circ$ There is no evidence that the isomeric product isomer is turning over to the desired product in these systems 


\section{$\underline{\text { Screen } 2}$}

Assess a wide range of palladium catalysts (32) with 3 methods to form palladium hydride species:

Method 1 (no additive) - In-house knowledge.

Method $2\left(\mathrm{Et}_{3} \mathrm{SiH}\right)$ - see Noonan, G. M.; Hayter, B. R.; Campbell, A. D.; Gorman, T. W.; Partridge, B. E.; Lamont, G. M. Tetrahedron Lett. 2013, 54, 4518-4521.

Method 3 (iPrCOCl) - see Gauthier, D.; Lindhardt, A. T.; Olsen, E. P. K.; Overgaard, J.; Skrydstrup, T. J. Am Chem. Soc. 2010, 132, 7998-8009.

\section{Experimental Set-Up}

- Reactions were performed in $1 \mathrm{~mL}$ vials in a 96-well plate format, situated in an inerted glovebox $\left(<10 \mathrm{ppm} \mathrm{O}_{2}\right.$ and $\left.<1 \mathrm{ppm} \mathrm{H}_{2} \mathrm{O}\right)$

- Ligands ( $12 \mathrm{~mol} \%$ for bidentate and $20 \mathrm{~mol} \%$ for monodentate) and Pd-113 were preweighed as solids using a Quantos weighing robot inside an inerted glovebox

- Pd sources (10mol\%), Internal Standard (IS, 4-4'-Di-tert-butyl biphenyl, 10mol\%) were dispensed as $0.01 \mathrm{M}$ stock solutions $\left(\mathrm{CHCl}_{3}\right)$ in the 96 -well vials according to reaction plan

- The carrier solvents were evaporated using a Genevac EZ-2 situated inside a glovebox

- Stir discs were added to each vial

- Three separate solutions of Alkene starting material (3 x 998mg in $20 \mathrm{~mL}$ toluene) were prepared:

- Vial 1 contained alkene in toluene

- Vial 2 contained alkene and $\mathrm{Et}_{3} \mathrm{SiH}(27.7 \mu \mathrm{L})$ was added to give $100 \mathrm{~mol} \%$ alkene and $10 \mathrm{~mol} \% \mathrm{Et}_{3} \mathrm{SiH}$ in each reaction

- Vial 3 contained alkene and $\mathrm{iPCOCl}(18.3 \mu \mathrm{L})$ was added to give $100 \mathrm{~mol} \%$ alkene and $10 \mathrm{~mol} \%$ iPrCOCl in each reaction

- $579 \mu \mathrm{L}$ of each solution was added in each vial to provide $28.9 \mathrm{mg}$ of alkene per reaction.

- $\mathrm{Et}_{3} \mathrm{~N}(1.4 \mu \mathrm{L}, 20 \mathrm{~mol} \%)$ was added to each vial that contained a ligand as its $\mathrm{HBF}_{4}$ salt (D4, D8 $\mathrm{D} 12$,E4, E8, E12, F4, F8, F12, G4, G8, G12)

- The reactions were sealed and heated at the $80^{\circ} \mathrm{C}$ for $20 \mathrm{~h}$

- Samples for UPLC/MS analysis were prepared at $2 \mathrm{~h}$ and $20 \mathrm{~h}$ 


\section{Reaction Plan}

\begin{tabular}{|c|c|c|c|c|c|c|c|c|c|c|c|c|}
\hline & \multicolumn{4}{|c|}{$10 \mathrm{~mol} \% \mathrm{Pd}(\mathrm{OAc}) 2$} & \multicolumn{4}{|c|}{$10 \mathrm{~mol} \% \mathrm{Pd}(\mathrm{OAc}) 2 / \mathrm{Et} 3 \mathrm{SiH}$ (10mol\%) } & \multicolumn{4}{|c|}{$10 \mathrm{~mol} \% \operatorname{Pd}(\mathrm{dba}) 2 / \mathrm{iPrCOCl}(10 \mathrm{~mol} \%)$} \\
\hline & 1 & 2 & 3 & 4 & 5 & 6 & 7 & 8 & 9 & 10 & 11 & 12 \\
\hline A & PPh3 & $\mathrm{P}(0$-tol)3 & $\mathrm{dppb}$ & dppp & PPh3 & $\mathrm{P}(\mathrm{o}$-tol)3 & $\mathrm{dppb}$ & dppp & PPh3 & $\mathrm{P}(0$-tol)3 & dppb & dppp \\
\hline B & $\begin{array}{c}\mathrm{P}(\mathrm{m}-(\mathrm{MeO})- \\
\mathrm{Ph}) 3\end{array}$ & $\begin{array}{c}\text { (Cy2- } \\
\text { naphOPO)PNM } \\
\text { e2 }\end{array}$ & dppm & Xantphos & $\begin{array}{c}\mathrm{P}(\mathrm{m} \text {-(MeO)- } \\
\mathrm{Ph}) 3\end{array}$ & $\begin{array}{c}\text { (Cy2- } \\
\text { naphOPO)PNM } \\
\text { e2 }\end{array}$ & dppm & Xantphos & $\begin{array}{c}\mathrm{P}(\mathrm{m}-(\mathrm{MeO})- \\
\mathrm{Ph}) 3\end{array}$ & $\begin{array}{c}\text { (Cy2- } \\
\text { naphOPO)PNM } \\
\text { e2 }\end{array}$ & dppm & Xantphos \\
\hline c & $\mathrm{P}(\mathrm{C6F5}) 3$ & $\mathrm{P}(\mathrm{OH})(\mathrm{t}-\mathrm{Bu}) 2$ & Phanephos & dpephos & $\mathrm{P}(\mathrm{CGF5}) 3$ & $\mathrm{P}(\mathrm{OH})(\mathrm{t}-\mathrm{Bu}) 2$ & Phanephos & dpephos & $\mathrm{P}(\mathrm{C6F5}) 3$ & $\mathrm{P}(\mathrm{OH})(\mathrm{t}-\mathrm{Bu}) 2$ & Phanephos & dpephos \\
\hline D & $\begin{array}{c}P(0-(2,4-t-B u)- \\
P h) 3\end{array}$ & $\mathrm{P}(\mathrm{Adam})_{2}(\mathrm{n}-\mathrm{Bu})$ & Biphephos & $\begin{array}{lll}P(t-B u) 3 . H B F 4 & \end{array}$ & $\begin{array}{c}P(0-(2,4-t-B u)- \\
P h) 3\end{array}$ & $P(\text { Adam })_{2}(n-B u)$ & Biphephos & P(t-Bu)3.HBF4 & $\begin{array}{c}\mathrm{P}(\mathrm{O}-(2,4-\mathrm{t}-\mathrm{Bu})- \\
\mathrm{Ph}) 3\end{array}$ & $\mathrm{P}(\mathrm{Adam})_{2}(\mathrm{n}-\mathrm{Bu})$ & Biphephos & $\mathrm{P}(\mathrm{t}-\mathrm{Bu}) 3 . \mathrm{HBF} 4$ \\
\hline E & Ru-phos & $\mathrm{P}\left(\mathrm{CH}_{2} \mathrm{CH}_{2} \mathrm{CN}\right)_{3}$ & $\begin{array}{c}1,3-(\mathrm{Di}- \\
\mathrm{tBu})_{2} \mathrm{PCH}_{2} \mathrm{Ph}\end{array}$ & $\begin{array}{c}\mathrm{P}(\mathrm{t}- \\
\mathrm{Bu})_{2}(\mathrm{Me}) \cdot \mathrm{HBF}_{4}\end{array}$ & Ru-phos & $\mathrm{P}\left(\mathrm{CH}_{2} \mathrm{CH}_{2} \mathrm{CN}\right)_{3}$ & $\begin{array}{c}1,3-(\mathrm{Di}- \\
\mathrm{tBu})_{2} \mathrm{PCH}_{2} \mathrm{Ph}\end{array}$ & $\begin{array}{c}\mathrm{P}(\mathrm{t}- \\
\mathrm{Bu})_{2}(\mathrm{Me}) \cdot \mathrm{HBF}_{4}\end{array}$ & Ru-phos & $\mathrm{P}\left(\mathrm{CH}_{2} \mathrm{CH}_{2} \mathrm{CN}\right)_{3}$ & $\begin{array}{c}1,3-(\mathrm{Di}- \\
\mathrm{tBu})_{2} \mathrm{PCH}_{2} \mathrm{Ph}\end{array}$ & $\begin{array}{c}\mathrm{P}(\mathrm{t}- \\
\mathrm{Bu})_{2}(\mathrm{Me}) \cdot \mathrm{HBF}_{4}\end{array}$ \\
\hline $\mathrm{F}$ & $\mathrm{P}(2$-furyl)3 & $\mathrm{P}(\mathrm{Ph})_{2}(\mathrm{C}-\mathrm{Hexyl})$ & BINAP & $\mathrm{PC}_{3} \cdot \mathrm{HBF} 4$ & $P(2$-furyl)3 & $\mathrm{P}(\mathrm{Ph})_{2}(\mathrm{c}-\mathrm{Hexyl})$ & BINAP & $\mathrm{PCY}_{3} \cdot \mathrm{HBF} 4$ & $P(2$-furyl)3 & $\mathrm{P}(\mathrm{Ph})_{2}(\mathrm{c}-\mathrm{Hexyl})$ & BINAP & $\mathrm{PC}_{3} . \mathrm{HBF} 4$ \\
\hline G & $\mathrm{P}(3,5-\mathrm{CF} 3 \mathrm{Ph}) 3$ & $x$-Phos & dppe & $\begin{array}{c:c}\mathrm{CV}_{2} \mathrm{P}_{(}\left(\mathrm{CH}_{2}\right)_{3} \mathrm{PCY}_{2} & \mathrm{HBF} 4\end{array}$ & F3Ph)3 & $x$-Phos & dppe & $\begin{array}{c}\mathrm{CC}_{2} \mathrm{P}\left(\mathrm{CH}_{2}\right)_{3} \mathrm{PCY}_{2} \\
\mathrm{HBF} 4\end{array}$ & CF3Ph)3 & 5 & dppe & $\begin{array}{c}\mathrm{C}_{2} \mathrm{P}_{2}\left(\mathrm{CH}_{2}\right)_{3} \mathrm{PCY}_{2} \\
\mathrm{HBF} 4\end{array}$ \\
\hline H & t-Bu-Xphos & dppf & DtBPF & $\mathrm{Pd}-113$ & $t-B u-X p h o s$ & dppf & DtBPF & $\mathrm{Pd}-113$ & $\mathrm{t}-\mathrm{Bu}$-Xphos & dppf & DtBPF & $\mathrm{Pd}-113$ \\
\hline
\end{tabular}


Results

Overview of results at $2 \mathrm{~h}$ (Product/Internal Standard values, higher values are higher yields)

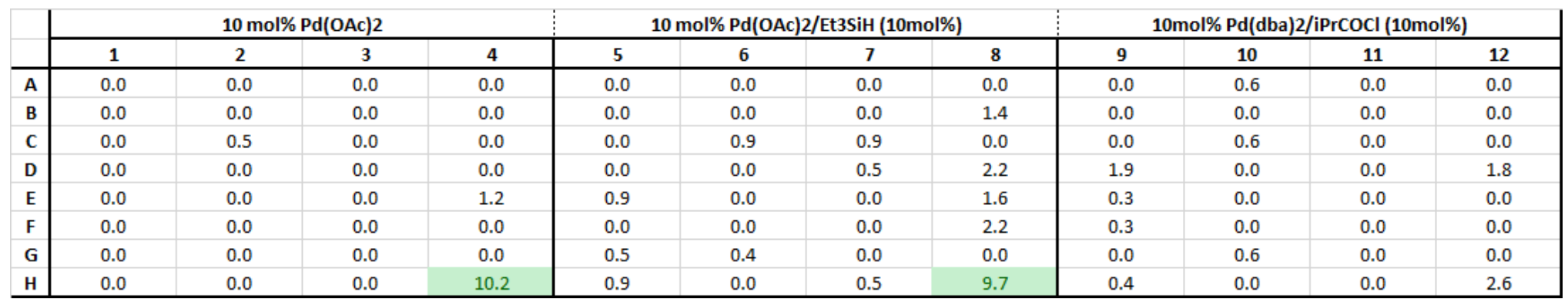

Overview of results at 20h (Product/Internal Standard values, higher values are higher yields)

\begin{tabular}{|c|c|c|c|c|c|c|c|c|c|c|c|c|}
\hline & \multicolumn{4}{|c|}{$10 \mathrm{~mol} \% \mathrm{Pd}(\mathrm{OAc}) 2$} & \multicolumn{4}{|c|}{$10 \mathrm{~mol} \% \mathrm{Pd}(\mathrm{OAC}) 2 / \mathrm{Et} 3 \mathrm{SiH}(10 \mathrm{~mol} \%)$} & \multicolumn{4}{|c|}{$10 \mathrm{~mol} \% \mathrm{Pd}(\mathrm{dba}) 2 / \mathrm{PrCOCl}$ (10mol\%) } \\
\hline & 1 & 2 & 3 & 4 & 5 & 6 & 7 & 8 & 9 & 10 & 11 & 12 \\
\hline A & 0.0 & 0.0 & 0.0 & 0.0 & 0.1 & 0.3 & 0.0 & 0.0 & 0.2 & 0.9 & 0.0 & 0.0 \\
\hline B & 0.0 & 0.0 & 0.0 & 0.9 & 0.0 & 0.0 & 0.0 & 1.3 & 0.2 & 0.0 & 0.0 & 0.0 \\
\hline C & 0.2 & 4.2 & 0.0 & 0.0 & 0.2 & 3.9 & 1.1 & 0.0 & 0.0 & 0.6 & 0.0 & 0.0 \\
\hline D & 0.0 & 0.0 & 0.4 & 0.6 & 0.4 & 0.0 & 0.7 & 1.3 & 1.5 & 0.0 & 0.0 & 2.1 \\
\hline E & 0.0 & 0.0 & 0.0 & 1.6 & 1.1 & 0.2 & 0.0 & 1.2 & 0.6 & 0.3 & 0.0 & 0.0 \\
\hline $\mathbf{F}$ & 0.0 & 0.0 & 0.0 & 0.0 & 0.0 & 0.0 & 0.0 & 1.0 & 0.3 & 0.0 & 0.0 & 0.0 \\
\hline G & 0.2 & 0.2 & 0.0 & 0.0 & 1.2 & 0.5 & 0.0 & 0.0 & 0.0 & 1.5 & 0.0 & 0.0 \\
\hline H & 0.0 & 0.0 & 0.0 & 8.8 & 1.5 & 0.0 & 0.4 & 9.1 & 0.4 & 0.1 & 0.0 & 3.4 \\
\hline
\end{tabular}


Results (sorted by product area\%)

\begin{tabular}{|c|c|c|c|c|c|c|c|c|c|c|c|c|c|c|c|c|c|c|c|c|}
\hline Row & $\begin{array}{c}\text { Col } \\
-\end{array}$ & $\begin{array}{l}\begin{array}{c}\text { Pd loading } \\
\text { (mol\%) }\end{array} \\
\end{array}$ & $\begin{array}{c}\begin{array}{c}\text { Metal Source } \\
\text { (10mol\%) }\end{array} \\
\end{array}$ & Ligand & $\begin{array}{l}\text { Ligand } \\
\text { charge } \\
\text { (mol\%) }\end{array}$ & $\begin{array}{r}\text { Additive } \\
\text { (10mol\%) }\end{array}$ & Et3N (mol\%) & $\begin{array}{c}\text { Alkene } \\
\text { (SM) }\end{array}$ & $\begin{array}{l}\text { Temp. } \\
\left({ }^{\circ} \mathrm{C}\right)\end{array}$ & Solvent & $\begin{array}{c}\text { Reaction } \\
\text { Conc. (vols) }\end{array}$ & Conv. (2h) & $\mathrm{P} / \mathrm{IS} \mathrm{(2h)}$ & Conv (20h) & $\mathrm{P} / \mathrm{IS}(20 \mathrm{~h})$ & Product & Alkene SM & $\begin{array}{r}\text { isomeric } \\
\text { product }\end{array}$ & SM - TBS & $\begin{array}{l}\text { Other } \\
\text { peaks }\end{array}$ \\
\hline $\mathrm{H}$ & 8 & 1.5 & $\begin{array}{ll}\text { Pd-113 } \\
\end{array}$ & none & 0 & Et3SiH & 0 & 1 eq. & 80 & Toluene & 20 & 98.3 & 9.7 & 93.5 & 9.1 & 85.3 & 0.0 & 5.9 & 0.4 & 8.4 \\
\hline $\mathrm{H}$ & 4 & 1.5 & $\mathrm{Pd}-113$ & none & 0 & none & 0 & 1 eq. & 80 & Toluene & 20 & 94.0 & 10.2 & 98.8 & 8.8 & 76.4 & 0.0 & 1.0 & 0.4 & 22.3 \\
\hline c & 6 & 1.5 & $\mathrm{Pd}(\mathrm{OAc}) 2$ & $\mathrm{P}(\mathrm{OH})(\mathrm{t}-\mathrm{Bu})_{2}^{2}$ & 20 & Et3SiH & 0 & 1 eq. & 80 & Toluene & 20 & 8.4 & 0.9 & 44.6 & 3.9 & 41.6 & 0.0 & 51.6 & 1.6 & 5.2 \\
\hline c & 2 & 1.5 & $\operatorname{Pd}(\mathrm{OAC}) 2$ & $\mathrm{P}(\mathrm{OH})(\mathrm{t}-\mathrm{Bu})_{2}^{2}$ & 20 & none & 0 & 1 eq. & 80 & Toluene & 20 & 5.1 & 0.5 & 40.2 & 4.2 & 38.4 & 52.2 & 4.9 & 0.0 & 4.5 \\
\hline H & 12 & 1.5 & Pd-113 & none & 0 & iPrCOCl & 0 & 1 eq. & 80 & Toluene & 20 & 96.1 & 2.6 & 97.3 & 3.4 & 34.4 & 0.0 & 1.0 & 1.4 & 63.3 \\
\hline D & 12 & 1.5 & $\mathrm{Pd}(\mathrm{dba}) 2$ & $\mathrm{P}(\mathrm{t}-\mathrm{Bu}) 3 . \mathrm{HBF} 4$ & 20 & iPrCOCl & 20 & 1 eq. & 80 & Toluene & 20 & 23.4 & 1.8 & 26.1 & 2.1 & 25.3 & 0.0 & 71.6 & 1.2 & 1.8 \\
\hline D & 8 & 1.5 & $\operatorname{Pd}(\mathrm{OAC}) 2$ & $\mathrm{P}(\mathrm{t}-\mathrm{Bu}) 3 . \mathrm{HBF} 4$ & 20 & Et3SiH & 20 & 1 eq. & 80 & Toluene & 20 & 23.1 & 2.2 & 25.0 & 1.3 & 24.5 & 0.0 & 73.5 & 0.0 & 2.0 \\
\hline $\mathrm{F}$ & 8 & 1.5 & $\mathrm{Pd}(\mathrm{OAC}) 2$ & $\mathrm{PCY}_{3} . \mathrm{HBF} 4$ & 20 & Et3SiH & 20 & 1 eq. & 80 & Toluene & 20 & 26.3 & 2.2 & 26.5 & 1.0 & 23.5 & 0.0 & 65.1 & 2.5 & 9.0 \\
\hline$E$ & 8 & 1.5 & $\mathrm{Pd}(\mathrm{OAC})_{2}^{2}$ & $\mathrm{P}(\mathrm{t}-\mathrm{Bu})_{2}(\mathrm{Me}) \cdot \mathrm{HBF}_{4}$ & 20 & Et3SiH & 20 & 1 eq. & 80 & Toluene & 20 & 17.0 & 1.6 & 21.3 & 1.2 & 20.0 & 0.7 & 73.1 & 0.0 & 6.2 \\
\hline $\mathrm{E}$ & 4 & 1.5 & $\mathrm{Pd}(\mathrm{OAC}) 2$ & $\mathrm{P}(\mathrm{t}-\mathrm{Bu})_{2}(\mathrm{Me}) \cdot \mathrm{HBF} F_{4}$ & 20 & none & 20 & 1 eq. & 80 & Toluene & 20 & 11.4 & 1.2 & 17.9 & 1.6 & 17.7 & 21.3 & 60.2 & 0.0 & 0.8 \\
\hline D & 9 & 1.5 & $\operatorname{Pd}(d b a) 2$ & $P(0-(2,4-4-B u)-P h) / 3$ & 20 & iPrCOCl & 0 & 1 eq. & 80 & Toluene & 20 & 25.9 & 1.9 & 27.1 & 1.5 & 14.3 & 0.0 & 38.3 & 6.1 & 41.3 \\
\hline $\mathrm{H}$ & 5 & 1.5 & $\mathrm{Pd}(\mathrm{OAC}) 2$ & t-Bu-Xphos & 20 & Et3SiH & 0 & 1 eq. & 80 & Toluene & 20 & 9.1 & 0.9 & 15.5 & 1.5 & 13.5 & 6.9 & 66.9 & 0.0 & 12.6 \\
\hline B & 8 & 1.5 & $\mathrm{Pd}(\mathrm{OAC}) 2$ & Xantphos & 12 & Et3SiH & 0 & 1 eq. & 80 & Toluene & 20 & 16.9 & 1.4 & 20.4 & 1.3 & 13.2 & 24.2 & 27.5 & 7.9 & 27.1 \\
\hline G & 10 & 1.5 & $\mathrm{Pd}(\mathrm{dba}) 2$ & $x$-Phos & 20 & iPrCOCl & 0 & 1 eq. & 80 & Toluene & 20 & 6.0 & 0.6 & 13.8 & 1.5 & 12.2 & 36.6 & 39.3 & 0.0 & 12.0 \\
\hline c & 7 & 1.5 & $\mathrm{Pd}(\mathrm{OAC})^{2}$ & Phanephos & 12 & Et3SiH & 0 & 1 eq. & 80 & Toluene & 20 & 11.1 & 0.9 & 13.4 & 1.1 & 10.1 & 1.3 & 63.7 & 3.5 & 21.4 \\
\hline $\mathrm{E}$ & 5 & 1.5 & $\mathrm{Pd}(\mathrm{OAC}) 2$ & Ru-phos & 20 & Et3SiH & 0 & 1 eq. & 80 & Toluene & 20 & 9.0 & 0.9 & 11.9 & 1.1 & 9.9 & 25.1 & 48.8 & 6.4 & 9.8 \\
\hline G & 5 & 1.5 & $\mathrm{Pd}(\mathrm{OAC})^{2}$ & $\mathrm{P}(3,5$-CF $3 \mathrm{Ph}) 3$ & 20 & Et3SiH & 0 & 1 eq. & 80 & Toluene & 20 & 4.5 & 0.5 & 12.3 & 1.2 & 9.7 & 69.3 & 0.1 & 7.1 & 13.8 \\
\hline B & 4 & 1.5 & $\mathrm{Pd}(\mathrm{OAC})^{2}$ & Xantphos & 12 & none & 0 & 1 eq. & 80 & Toluene & 20 & 0.0 & 0.0 & 10.3 & 0.9 & 8.5 & 59.6 & 14.2 & 6.6 & 11.2 \\
\hline A & 10 & 1.5 & $\mathrm{Pd}(\mathrm{dba}) 2$ & $P(0-00) 3$ & 20 & iPrCOCl & 0 & 1 eq. & 80 & Toluene & 20 & 8.5 & 0.6 & 12.8 & 0.9 & 7.7 & 15.1 & 37.7 & 5.6 & 33.8 \\
\hline$E$ & 9 & 1.5 & $\mathrm{Pd}(\mathrm{dba})^{2}$ & Ru-phos & 20 & iPrCOCl & 0 & 1 eq. & 80 & Toluene & 20 & 3.5 & 0.3 & 7.2 & 0.6 & 6.7 & 54.9 & 32.1 & 0.0 & 6.3 \\
\hline D & 7 & 1.5 & $\mathrm{Pd}(\mathrm{OAC}) 2$ & Biphephos & 12 & Et3SiH & 0 & $1 \mathrm{eq}$. & 80 & Toluene & 20 & 5.8 & 0.5 & 10.9 & 0. & 5.7 & 39.5 & 6.7 & 12.4 & 35.8 \\
\hline D & 4 & 1.5 & $\mathrm{Pd}(\mathrm{OAC}) 2$ & $P(t-B u) 3 . H B F 4$ & 20 & none & 20 & $1 \mathrm{eq}$. & 80 & Toluene & 20 & 0.0 & 0.0 & 5.4 & 0.6 & 5.2 & 76.4 & 14.3 & 1.6 & 2.5 \\
\hline
\end{tabular}

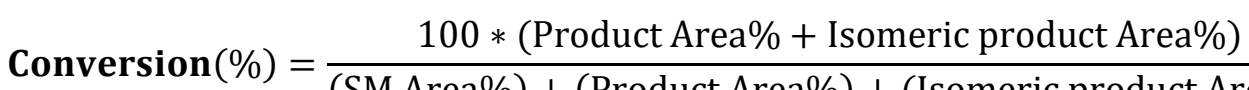

$=\frac{1 \text { (SM Area } \%)+(\text { Product Area } \%)+(\text { Isomeric product Area } \%)}{(\text { ) }}$ 
Reaction Products and Impurities

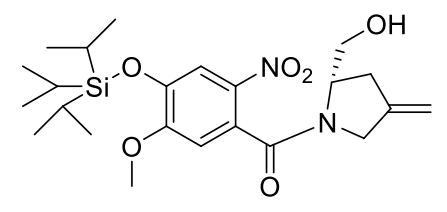

SM - TBS, Rt=1.09mins Molecular Weight: 464.63

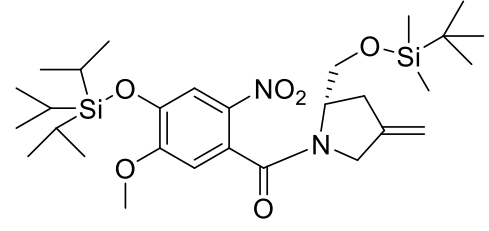

Starting material, Rt=7.9mins Molecular Weight: 578.89

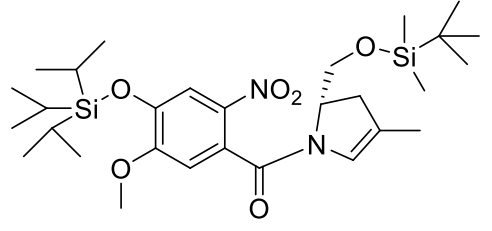

Product, $\mathrm{Rt}=8.2 \mathrm{mins}$ Molecular Weight: 578.89

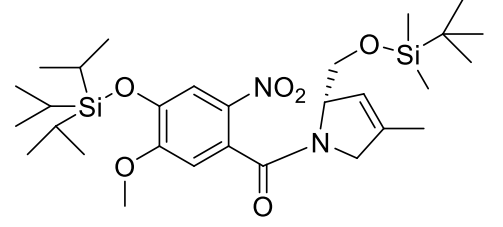

Isomeric product, Rt=7.6mins Molecular Weight: 578.89

Typical HPLCs (toluene peak at $0.5 \mathrm{mins}$ )

Reaction $\mathrm{H} 4\left(\mathrm{Pd}-113\right.$, toluene, $80^{\circ} \mathrm{C}, 2 \mathrm{~h}$ )

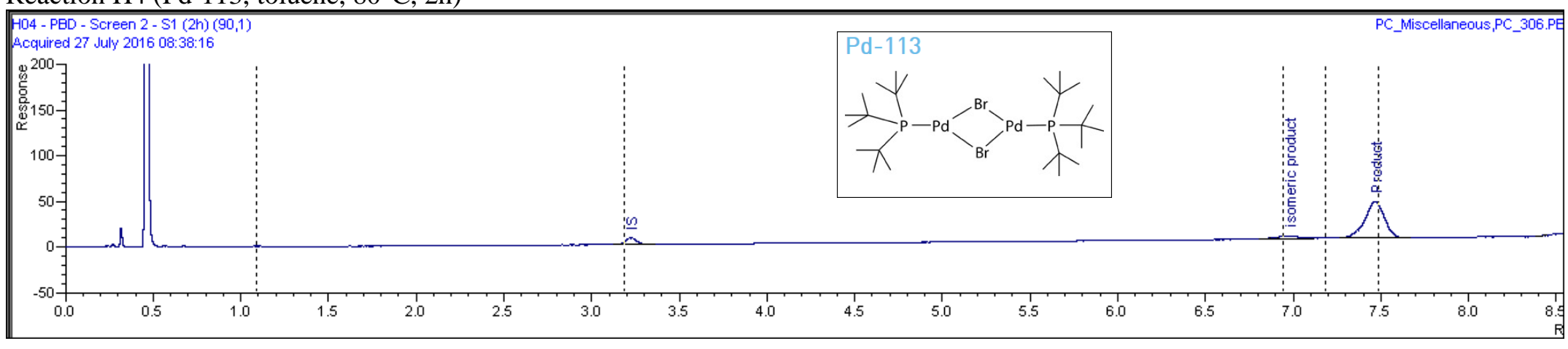

Reaction H8 (Pd-113/Et 3 SiH (10mol\%), toluene, 80C, 2h)

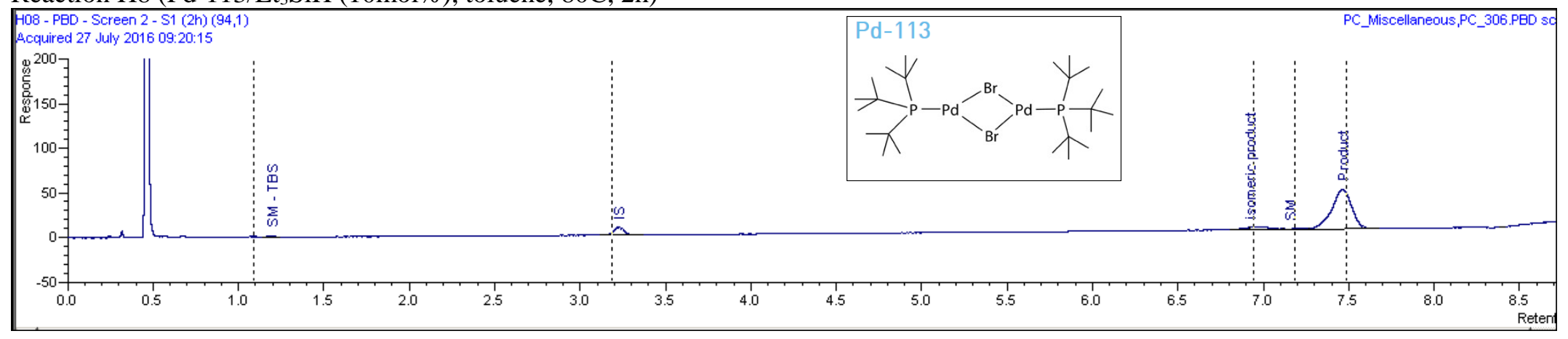




\section{Conclusions}

- No other catalyst other than Pd-113 appears to work well to give clean conversion to product

- The addition of $\mathrm{Et}_{3} \mathrm{SiH}$ appears to avoid a Pd-mirror on the reaction vial 


\section{Screen 3}

Isomerisation screen focussing on understanding the mechanism and potential for scale up utilising $\mathrm{P}^{\mathrm{t}} \mathrm{Bu}_{3}$ as a ligand in various different palladium combinations

\section{Experimental Set-Up}

- On a $100 \mathrm{mg}$ scale of alkene, reactions were performed in $4 \mathrm{~mL}$ vials in a 24 -well plate format, situated in an inerted glovebox $\left(<10 \mathrm{ppm}_{2}\right.$ and <1ppm $\left.\mathrm{H}_{2} \mathrm{O}\right)$.

- Grubbs II, benzoquinone and product and internal standard (for reaction 14 only) were weighed by hand and the vials then placed into the glovebox environment.

- All other solids were dispensed as solids using a Quantos weighing robot situated inside the glovebox.

- Stir discs were added to each vial.

- Starting material (1.4g) and internal standard (64.4mg) was dissolved in dry degassed toluene (14mL total) and $1 \mathrm{~mL}$ was dispensed to each vial. (100mg/reaction and $10 \mathrm{~mol} \%$ internal standard)

- Reaction 14 contained product (100mg), internal standard (4.6mg, 10mol\%) and $1 \mathrm{~mL}$ of toluene was added.

- Liquid additives for reactions 5, 6, 10 and 11 were then added.

- The reactions were sealed and heated at the $70^{\circ} \mathrm{C}$ for $18 \mathrm{~h}$ for all reactions.

- Samples for UPLC/MS analysis were prepared at $1 \mathrm{~h}$ and $22 \mathrm{~h}$. 
Reaction Plan and Results

\begin{tabular}{|c|c|c|c|c|c|c|c|c|c|c|c|c|c|c|c|c|c|c|}
\hline & & & & & & & & & & & & & & \multicolumn{5}{|c|}{ Area\% at $220 \mathrm{nM}$ at $18 \mathrm{~h}$} \\
\hline $\begin{array}{l}\text { Reaction } \\
\text { Number }\end{array}$ & Catalyst & $\begin{array}{l}\text { Catalyst } \\
\text { (mol\%) }\end{array}$ & Additive & $\begin{array}{l}\text { Additive } \\
\text { (mol\%) }\end{array}$ & Alkene (SM) & Temp. ${ }^{\circ} \mathrm{C}$ & $\begin{array}{l}\text { Solvent } \\
\text { (10vols) }\end{array}$ & $\begin{array}{l}\text { Product } \\
\text { (Area\%) } \\
\text { at } 0.5 \mathrm{~h}\end{array}$ & $\begin{array}{c}\text { SM } \\
\text { (Area\%) } \\
\text { at } 0.5 \mathrm{~h}\end{array}$ & $\begin{array}{c}\text { Product } \\
\text { isomer } \\
\text { (Area\%) } \\
\text { at } 0.5 \mathrm{~h}\end{array}$ & $\begin{array}{c}\text { Product } \\
\text { (Area\%) } \\
\text { at 2h }\end{array}$ & $\begin{array}{c}\text { SM } \\
\text { (Area\%) } \\
\text { at } 2 \mathrm{~h}\end{array}$ & $\begin{array}{c}\text { Product } \\
\text { isomer } \\
\text { (Area\%) } \\
\text { at 2h }\end{array}$ & $\begin{array}{c}\text { Product } \\
/ \text { IS at } \\
18 \mathrm{~h}\end{array}$ & $\begin{array}{c}\text { Product } \\
\text { (Area\%) } \\
\text { at 18h }\end{array}$ & $\begin{array}{c}\text { SM } \\
\text { (Area\%) } \\
\text { at } 18 \mathrm{~h}\end{array}$ & $\begin{array}{c}\text { Product } \\
\text { isomer } \\
\text { (Area\%) } \\
\text { at 18h } \\
\end{array}$ & $\begin{array}{l}\text { Other } \\
\text { peaks }\end{array}$ \\
\hline 1 & Grubbs II & 5 & n-BuOH & 1000uL & 1.0 eq. SM & 70 & Toluene & 23.3 & 53.8 & 20.7 & 45.3 & 6.9 & 47.1 & 5.5 & 54.2 & 0.0 & 44.1 & 1.7 \\
\hline 2 & Pd-113 & 5 & none & none & 1.0eq. SM & 70 & Toluene & 89.4 & 0.0 & 7.5 & 92.4 & 0.0 & 7.6 & 12.9 & 89.1 & 0.0 & 6.3 & 4.5 \\
\hline 3 & Pd-113 & 2.5 & none & none & 1.0eq. SM & 70 & Toluene & 82.5 & 0.0 & 16.1 & 90.3 & 0.0 & 8.9 & 13.0 & 89.2 & 0.0 & 8.2 & 2.6 \\
\hline 4 & Pd-113 & 1.25 & none & none & 1.0eq. SM & 70 & Toluene & 64.0 & 0.0 & 35.8 & 82.9 & 0.0 & 16.8 & 11.7 & 82.8 & 0.0 & 16.0 & 1.2 \\
\hline 5 & Pd-113 & 2.5 & Et3SiH & 10 & 1.0eq. SM & 70 & Toluene & 68.0 & 0.0 & 25.4 & 88.3 & 0.0 & 10.7 & 11.7 & 87.2 & 0.0 & 5.5 & 7.3 \\
\hline 6 & Pd-113 & 2.5 & Et3N & 20 & 1.0eq. SM & 70 & Toluene & 21.0 & 0.0 & 78.4 & 20.7 & 0.0 & 78.8 & 2.7 & 20.6 & 0.0 & 78.8 & 0.6 \\
\hline 7 & Pd-113 & 2.5 & PPh3 & 10 & 1.0eq. SM & 70 & Toluene & 15.0 & 41.1 & 43.6 & 15.7 & 37.7 & 46.2 & 2.3 & 17.1 & 32.9 & 49.7 & 0.3 \\
\hline 8 & Pd-113 & 2.5 & Benzoquinone & 10 & 1.0eq. SM & 70 & Toluene & 0.0 & 98.7 & 0.2 & 1.7 & 97.2 & 0.3 & 0.2 & 1.6 & 95.9 & 1.8 & 0.7 \\
\hline 9 & $\mathrm{Pd}-113$ & 2.5 & Phenanthroline & 5 & 1.0eq. SM & 70 & Toluene & 0.0 & 99.4 & 0.2 & 0.0 & 99.5 & 0.1 & 0.0 & 0.0 & 99.7 & 0.2 & 0.2 \\
\hline 10 & $\mathrm{Pd}(\mathrm{OAc}) 2$ & 5 & tBu3P/Et3SiH & 5,10 & 1.0 eq. SM & 70 & Toluene & 0.0 & 98.0 & 0.1 & 2.5 & 92.9 & 0.2 & 0.6 & 4.4 & 79.9 & 10.3 & 5.5 \\
\hline 11 & $\mathrm{Pd}(\mathrm{OAc}) 2$ & 5 & tBu3P/Et3SiH & 10,10 & 1.0eq. SM & 70 & Toluene & 0.0 & 99.6 & 0.2 & 0.0 & 98.7 & 0.1 & 0.0 & 0.0 & 98.3 & 0.2 & 1.6 \\
\hline 12 & (tBu3P) $2 \mathrm{Pd}(\mathrm{HCl})$ & 5 & none & none & 1.0 eq. SM & 70 & Toluene & 37.2 & 0.0 & 62.0 & 47.1 & 0.0 & 52.5 & 7.8 & 59.5 & 0.0 & 40.0 & 0.5 \\
\hline 13 & (tBu3P) $2 \mathrm{Pd}(\mathrm{HCl})$ & 2.5 & none & none & 1.0eq. SM & 70 & Toluene & 45.6 & 0.0 & 53.6 & 57.5 & 0.0 & 42.1 & 10.0 & 75.0 & 0.0 & 24.7 & 0.3 \\
\hline 14 & Pd-113 & 5 & none & none & 1.0eq produc & 70 & Toluene & 94.5 & 0.0 & 5.5 & 92.4 & 0.0 & 7.6 & 13.1 & 90.5 & 0.0 & 7.5 & 2.1 \\
\hline
\end{tabular}


Reaction products and impurities

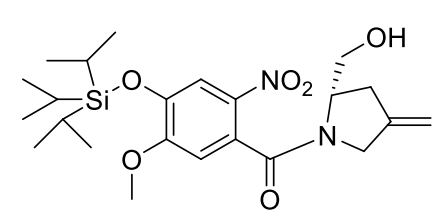

SM - TBS, Rt=1.09mins Molecular Weight: 464.63

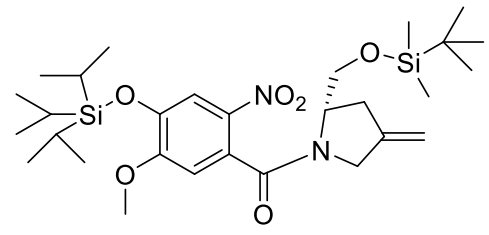

Starting material, $\mathbf{R t}=7.9 \mathrm{mins}$ Molecular Weight: 578.89

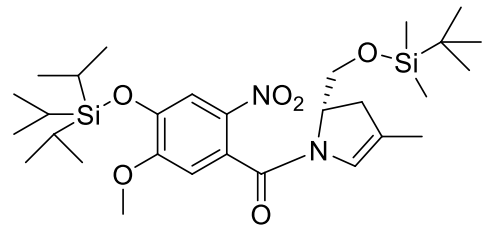

Product, $\mathrm{Rt}=8.2 \mathrm{mins}$

Molecular Weight: 578.89

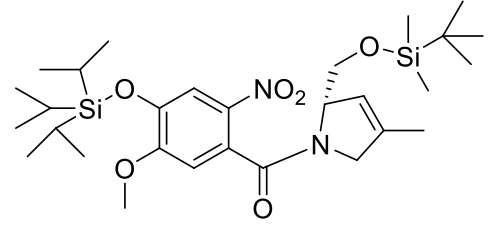

Isomeric product, $\mathbf{R t}=7.6 \mathrm{mins}$ Molecular Weight: 578.89

Typical HPLCs (toluene peak at $0.5 \mathrm{mins}$ )

Reaction 4 (Pd-113 (1.25mol\%), Toluene, $\left.70^{\circ} \mathrm{C}, 18 \mathrm{~h}\right)$

A04 (R4) - PED - Screen 3 - S3 (18h) (36,1)

PC_Miscellaneous, $\mathrm{PC} \_306 . \mathrm{PBD}$ sCl
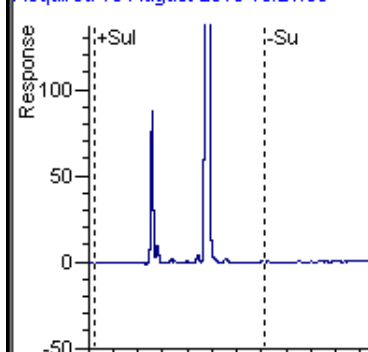

(18,

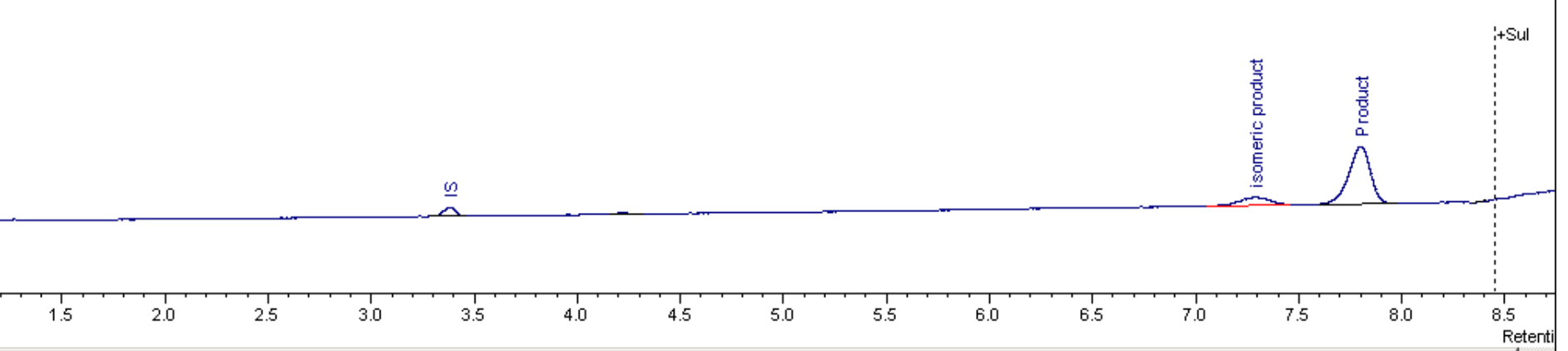

Reaction $3\left(\mathrm{Pd}-113(2.5 \mathrm{~mol} \%)\right.$, Toluene, $\left.70^{\circ} \mathrm{C}, 18 \mathrm{~h}\right)$

AO03 (R3) - PED - Screen 3 - S3(18h) (35,1)
Acquired 18 August 2016 10:11:27

(1)
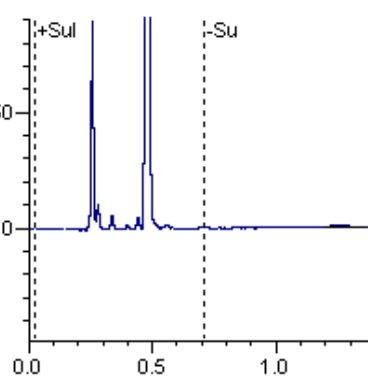

PC_Miscellaneous,PC_306.PBD so

0.5

1.5 2.0 2.5 $\begin{array}{ll}8.0 & 8.5 \\ & \text { Retent }\end{array}$ 
Reaction 14 (Pd-113 (5mol\%), Toluene, $70^{\circ} \mathrm{C}, 18 \mathrm{~h}$, no starting material added, only product)

C02 (R14) - PED - Screen $3-3$ S (18h) (46,1)

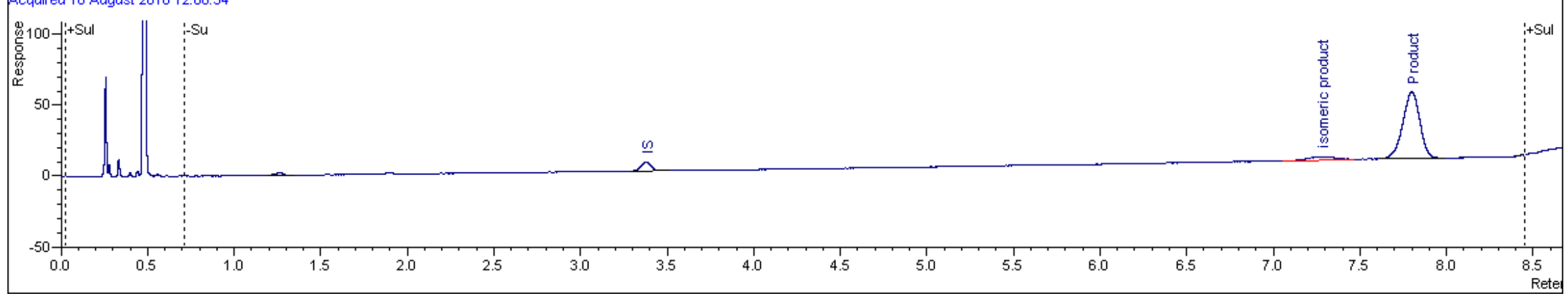

Reaction 5 (Pd-113 (2.5mol\%), $\mathrm{Et}_{3} \mathrm{SiH}(10 \mathrm{~mol} \%)$ Toluene, $\left.70^{\circ} \mathrm{C}, 18 \mathrm{~h}\right)$

4.05 (R5) - PBD - Screen 3 - S3 (18h) (37,1)

4.05 (R5) - PED - Screen 3 - S3 (12

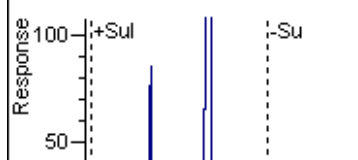
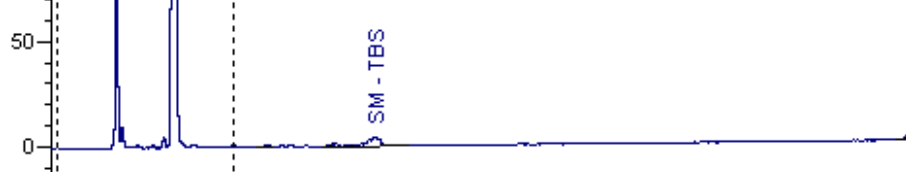

PC Miscellaneous, PC 306. PED

:

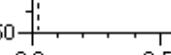

$0.5+1.0$

$1.5+2.0$

2.5

3.0

3.5

4.0

4.5

$5.0^{1}$

5.5

6.0

6.5

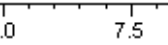




\section{Conclusions}

- Alternative methods to generate $\mathrm{tBu}_{3} \mathrm{PPd}(\mathrm{HX})$ do not work as well as Pd-113 e.g. reactions 10,11

- Grubbs catalyst derived Ru-H catalysts do not reach full conversion. (Reaction 1)

- Co-ligand addition (Reactions 6-9) prevent mirroring but also stall the reaction.

- Use of isolated palladium complex $\left(\mathrm{tBu}_{3} \mathrm{P}\right)_{2} \mathrm{PdHCl}$ (see Hills, I. D.; Fu, G. C. J. Am. Chem. Soc., 2004, 126, 13178-13179 for method of preparation) is less effective than Pd-113.

- The reaction proceeds smoothly with Pd-113 even with loading as low as $1.25 \mathrm{~mol} \%$ to afford $80 \%$ product (area\%)

- Addition of $\mathrm{Et}_{3} \mathrm{SiH}(10 \mathrm{~mol} \%)$ appears to avoid Pd-mirroring (Reaction 5)

- If the product is submitted to the reaction conditions (Reaction 14) the reaction again ends up with the equilibrium ratio of products. 


\section{$\underline{\text { Reaction Profiling }}$}

Preparation of (S)-(2-amino-5-methoxy-4-((triisopropylsilyl)oxy)phenyl)(2-(((tertbutyldimethylsilyl)oxy)methyl)-4-methyl-2,3-dihydro-1H-pyrrol-1-yl)methanone (6)

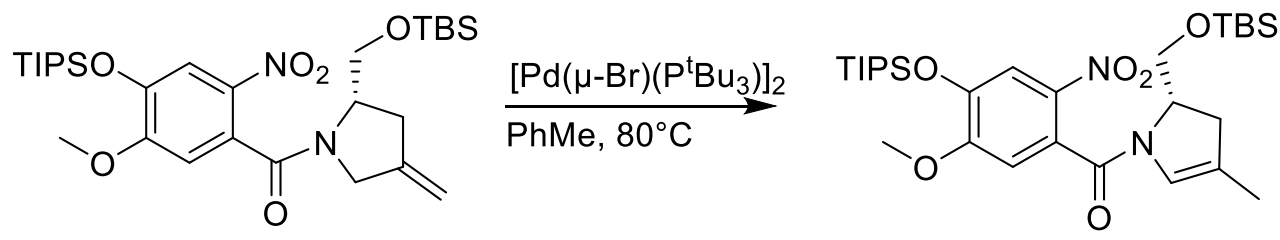

To an inerted solution of $(S)$-(2-(((tert-butyldimethylsilyl)oxy)methyl)-4-methylenepyrrolidin-1-yl)(5methoxy-2-nitro-4-((triisopropylsilyl)oxy)phenyl)methanone $(8,50 \mathrm{mg}, 100.0 \% \mathrm{w} / \mathrm{w}, 861 \mu \mathrm{mol})$ in toluene $(1 \mathrm{~mL})$ was added di- $\mu$-bromobis(tri-tert-butylphosphine)dipalladium(I) $(3.34 \mathrm{mg}, 4.32 \mu \mathrm{mol}$, $0.05 \mathrm{eq}$ ). The reaction was heated to $80^{\circ} \mathrm{C}$ and sampled regularly.

\section{UPLC Analysis:}

Column: Phenomenex Kinetex 2.6u C18 100A 75mm x 3mm

Mobile phase A: $0.03 \%$ TFA in water

Mobile phase B: $0.025 \%$ TFA in acetonitrile

Gradient:

\begin{tabular}{|l|l|l|}
\hline Time & $\% \mathrm{~A}$ & $\% \mathrm{~B}$ \\
\hline 0.00 & 30 & 70 \\
\hline 8.00 & 15 & 85 \\
\hline 8.50 & 5 & 95 \\
\hline
\end{tabular}

Flow rate: $1.2 \mathrm{~mL} / \mathrm{min}$

Detection: UV @ 220nm

Temperature: $40^{\circ} \mathrm{C}$

\section{Profile Table}

\begin{tabular}{|l|l|l|l|}
\hline Time & $\begin{array}{l}\text { Starting } \\
\text { Material }(\mathbf{8})\end{array}$ & Isomer $(\mathbf{1 1})$ & Product $(\mathbf{6})$ \\
\hline Minutes & Area $\%$ & Area $\%$ & Area $\%$ \\
\hline 0 & 100 & 0 & 0 \\
\hline 1 & 35 & 51 & 13 \\
\hline 2 & 0 & 81 & 19 \\
\hline 5 & 0 & 78 & 22 \\
\hline 10 & 0 & 73 & 27 \\
\hline 20 & 0 & 43 & 57 \\
\hline 60 & 0 & 16 & 84 \\
\hline 120 & 0 & 12 & 88 \\
\hline 200 & 0 & 10 & 90 \\
\hline 310 & 0 & 10 & 90 \\
\hline 1320 & 0 & 9 & 91 \\
\hline
\end{tabular}




\section{Profile Graph}

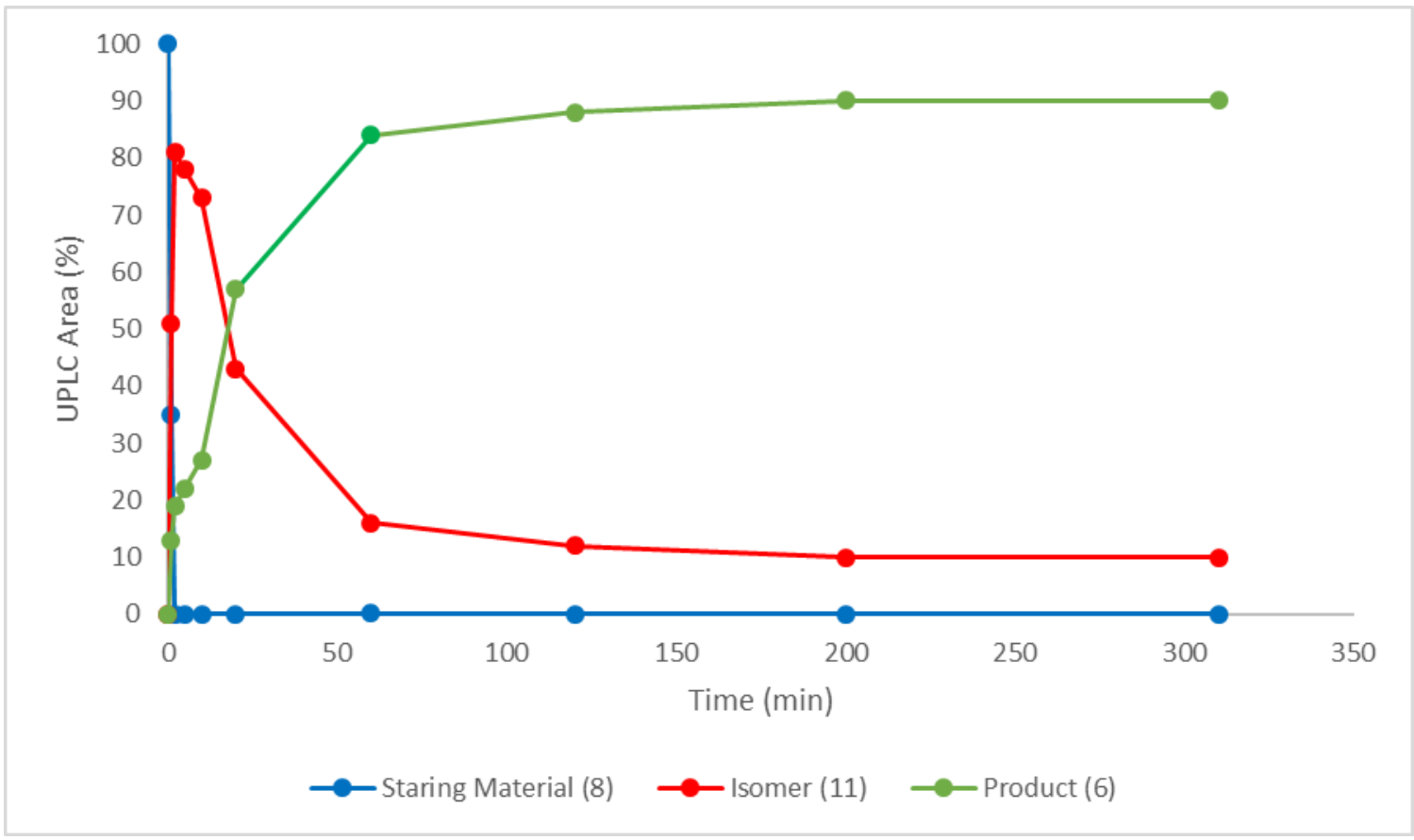




\section{4-Methyl-2,5-dihydropyrrole (11) and 2-Methylene-4-methylpyrrolidine (12) Isomers}

Isomerisation of (S)-(2-(((tert-butyldimethylsilyl)oxy)methyl)-4-methylenepyrrolidin-1-yl)(5-methoxy2-nitro-4-((triisopropylsilyl)oxy)phenyl)methanone (8)

To an inerted solution of (S)-(2-((tert-butyldimethylsilyl)oxy)methyl)-4-methylenepyrrolidin-1-yl)(5methoxy-2-nitro-4-((triisopropylsilyl)oxy)phenyl)methanone $(\mathbf{8}, 296 \mathrm{mg}, 100.0 \% \mathrm{w} / \mathrm{w}, 0.551 \mathrm{mmol})$ in toluene $(3 \mathrm{~mL})$ was added di- $\mu$-bromobis(tri-tert-butylphosphine)dipalladium(I) $(9.94 \mu \mathrm{g}, 12.4 \mu \mathrm{mol}$, $0.025 \mathrm{eq}$ ). The reaction was heated to $70^{\circ} \mathrm{C}$ and after 2 hours in-process analysis indicated the isomerisation had stalled at $73 \%$ isomer and $26 \%$ product. The reaction mixture was cooled, filtered and solvent removed in vacuo affording a yellow oil. The mixture was purified using flash column chromatography (25g of silica gel, gradient of $0 \%$ to $100 \%$ MTBE in heptane) affording:

(S)-(2-(((tert-butyldimethylsilyl)oxy)methyl)-4-methyl-2,5-dihydro-1H-pyrrol-1-yl)(5-methoxy-2-nitro4-((triisopropylsilyl)oxy)phenyl)methanone (11)<smiles>CCCCO[C@]1(OC(C)(C)C)C=C(C)CN1C(=O)c1cc(OC)c(OC)cc1[N+](=O)[O-]</smiles>

Pale yellow oil

\section{Major Rotamer (75\%):}

${ }^{1} \mathrm{H}$ NMR $\left(500 \mathrm{MHz}, \mathrm{CDCl}_{3}\right) 0.08(\mathrm{~d}, \mathrm{~J}=6.6 \mathrm{~Hz}, 6 \mathrm{H}), 0.90(\mathrm{~s}, 9 \mathrm{H}), 1.11(\mathrm{dt}, \mathrm{J}=7.5,1.3 \mathrm{~Hz}, 18 \mathrm{H})$, $1.28(\mathrm{ddd}, \mathrm{J}=14.8,7.4,2.6 \mathrm{~Hz}, 3 \mathrm{H}), 1.69(\mathrm{t}, \mathrm{J}=1.6 \mathrm{~Hz}, 3 \mathrm{H}), 3.64-3.83(\mathrm{~m}, 2 \mathrm{H}), 3.90(\mathrm{~s}, 3 \mathrm{H}), 3.92-$ $4.09(\mathrm{~m}, 2 \mathrm{H}), 4.96(\mathrm{~s}, 1 \mathrm{H}), 5.54(\mathrm{~s}, 1 \mathrm{H}), 6.77(\mathrm{~s}, 1 \mathrm{H}), 7.70(\mathrm{~s}, 1 \mathrm{H})$.

${ }^{13} \mathrm{C}$ NMR (126 MHz, $\left.\mathrm{CDCl}_{3}\right)$ 166.00, 156.10, 145.44, 137.00, 134.45, 127.89, 122.77, 115.77, 109.33, $65.81,62.47,58.29,55.75,25.53,17.85,17.51,13.79,12.49,-5.64$.

\section{Minor Rotamer (25\%):}

${ }^{1} \mathrm{H}$ NMR $\left(500 \mathrm{MHz}, \mathrm{CDCl}_{3}\right) 0.08(\mathrm{~d}, \mathrm{~J}=6.6 \mathrm{~Hz}, 6 \mathrm{H}), 0.90(\mathrm{~s}, 9 \mathrm{H}), 1.11(\mathrm{dt}, \mathrm{J}=7.5,1.3 \mathrm{~Hz}, 18 \mathrm{H})$, 1.28 (ddd, $\mathrm{J}=14.8,7.4,2.6 \mathrm{~Hz}, 3 \mathrm{H}), 1.69(\mathrm{t}, \mathrm{J}=1.6 \mathrm{~Hz}, 3 \mathrm{H}), 3.64-3.83(\mathrm{~m}, 2 \mathrm{H}), 3.90(\mathrm{~s}, 3 \mathrm{H}), 3.92-$ $4.09(\mathrm{~m}, 2 \mathrm{H}), 4.54(\mathrm{~d}, \mathrm{~J}=15.2 \mathrm{~Hz}, 1 \mathrm{H}), 5.38(\mathrm{~s}, 1 \mathrm{H}), 6.82(\mathrm{~s}, 1 \mathrm{H}), 7.71(\mathrm{~s}, 1 \mathrm{H})$.

(E)-(2-(((tert-butyldimethylsilyl)oxy)methylene)-4-methylpyrrolidin-1-yl)(5-methoxy-2-nitro-4((triisopropylsilyl)oxy)phenyl)methanone (12)<smiles>COc1cc(C(=O)N2C[C@H](C)CC2=CO[SnH3])c([N+](=O)[O-])cc1OC</smiles>

Pale yellow oil

${ }^{1} \mathrm{H}$ NMR $\left(500 \mathrm{MHz}, \mathrm{CDCl}_{3}\right) 0.18(\mathrm{~d}, \mathrm{~J}=0.7 \mathrm{~Hz}, 6 \mathrm{H}), 0.96(\mathrm{~s}, 9 \mathrm{H}), 1.00(\mathrm{~d}, \mathrm{~J}=6.5 \mathrm{~Hz}, 3 \mathrm{H}), 1.11(\mathrm{~d}, \mathrm{~J}$ $=7.4 \mathrm{~Hz}, 18 \mathrm{H}), 1.22-1.35(\mathrm{~m}, 3 \mathrm{H}), 2.21-2.34(\mathrm{~m}, 2 \mathrm{H}), 2.88-2.98(\mathrm{~m}, 2 \mathrm{H}), 3.37(\mathrm{dd}, \mathrm{J}=10.0,6.5$ $\mathrm{Hz}, 1 \mathrm{H}), 3.91$ (s, 3H), $6.76(\mathrm{~s}, 1 \mathrm{H}), 7.70(\mathrm{~s}, 1 \mathrm{H}), 7.83$ (s, 1H).

${ }^{13} \mathrm{C}$ NMR $\left(126 \mathrm{MHz}, \mathrm{CDCl}_{3}\right)$ 163.65, 156.12, 145.38, 136.68, 129.99, 128.35, 127.35, 115.66, 109.21, 
NMR assignment of $\mathbf{1 1}$ was complicated by the presence of rotamers, thus reduction of the nitro group was completed to afford the aniline:

Preparation of (S)-(2-amino-5-methoxy-4-((triisopropylsilyl)oxy)phenyl)(2-(((tertbutyldimethylsilyl)oxy)methyl)-4-methyl-2,5-dihydro-1H-pyrrol-1-yl)methanone<smiles>COc1cc(C(=O)N2CC(C)=C[C@H]2CO[SbH2])c([N+](=O)[O-])cc1OC</smiles><smiles>CCOC(=O)COCCO</smiles><smiles>COc1cc(N)c(C(=O)N2CC(C)=C[C@H]2CO[AsH3])cc1OC</smiles>

Zinc $(170 \mathrm{mg}, 2.60 \mathrm{mmol})$ was added to a mixture of ethanol $(0.32 \mathrm{~mL})$, water $(0.020 \mathrm{~mL})$ and formic acid $(0.020 \mathrm{~mL}, 0.53 \mathrm{mmol})$ at ambient temperature and stirred vigorously. To this mixture, a solution $(S)$ (2-(((tert-butyldimethylsilyl)oxy)methyl)-4-methyl-2,5-dihydro-1H-pyrrol-1-yl)(5-methoxy-2-nitro-4((triisopropylsilyl)oxy)phenyl)methanone $(\mathbf{1 1}, 20 \mathrm{mg}, 0.0345 \mathrm{mmol})$ in ethanol $(0.080 \mathrm{~mL})$ was added dropwise with a pipette. The reaction was stirred for $30 \mathrm{~min}$ at room temperature at which time inprocess analysis indicated the reaction was complete. The mixture was diluted with ethyl acetate $(3 \mathrm{~mL})$, the solids removed by filtration through a plug of cotton wool and the filtrate was washed with saturated sodium bicarbonate solution $(5 \mathrm{~mL})$. The volatiles were removed by evaporation to give the product (17 $\mathrm{mg}, 0.0310 \mathrm{mmol}, 90 \%$ ) as a colourless gummy solid.

${ }^{1} \mathrm{H}$ NMR (500 MHz, $\left.\mathrm{CDCl}_{3}\right)-0.04-0.1(\mathrm{~m}, 6 \mathrm{H}), 0.87(\mathrm{~s}, 9 \mathrm{H}), 1.07-1.12(\mathrm{~m}, 18 \mathrm{H}), 1.19-1.31(\mathrm{~m}$, $3 \mathrm{H}), 1.73(\mathrm{~s}, 3 \mathrm{H}), 3.71(\mathrm{~s}, 3 \mathrm{H}), 3.78(\mathrm{~s}, 1 \mathrm{H}), 3.81-4.05(\mathrm{~m}, 2 \mathrm{H}), 4.23-4.32(\mathrm{~m}, 2 \mathrm{H}), 5.37-5.45(\mathrm{~m}$, $1 \mathrm{H}), 6.26(\mathrm{~s}, 1 \mathrm{H}), 6.77(\mathrm{~s}, 1 \mathrm{H})$.

${ }^{13} \mathrm{C}$ NMR (126 MHz, $\left.\mathrm{CDCl}_{3}\right)$ 169.25, 148.10, 142.74, 140.19, 135.46, 121.85, 113.05, 112.83, 108.94, $65.55,62.82,59.46,56.28,25.50,17.86,17.63,13.83,12.60,-5.69$. 
(S)-(2-(((tert-butyldimethylsilyl)oxy)methyl)-4-methyl-2,5-dihydro-1H-pyrrol-1-yl)

(5-methoxy-2-nitro-4-((triisopropylsilyl)oxy)phenyl)methanone (11)

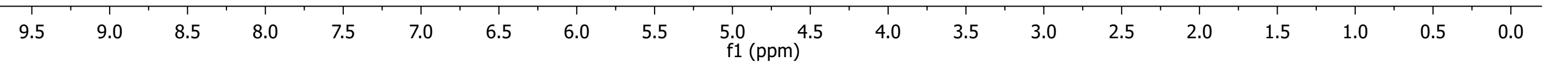


(S)-(2-(((tert-butyldimethylsilyl)oxy)methyl)-4-methyl-2,5-dihydro-1H-pyrrol-1-yl) (5-methoxy-2-nitro-4-((triisopropylsilyl)oxy)phenyl)methanone (11)

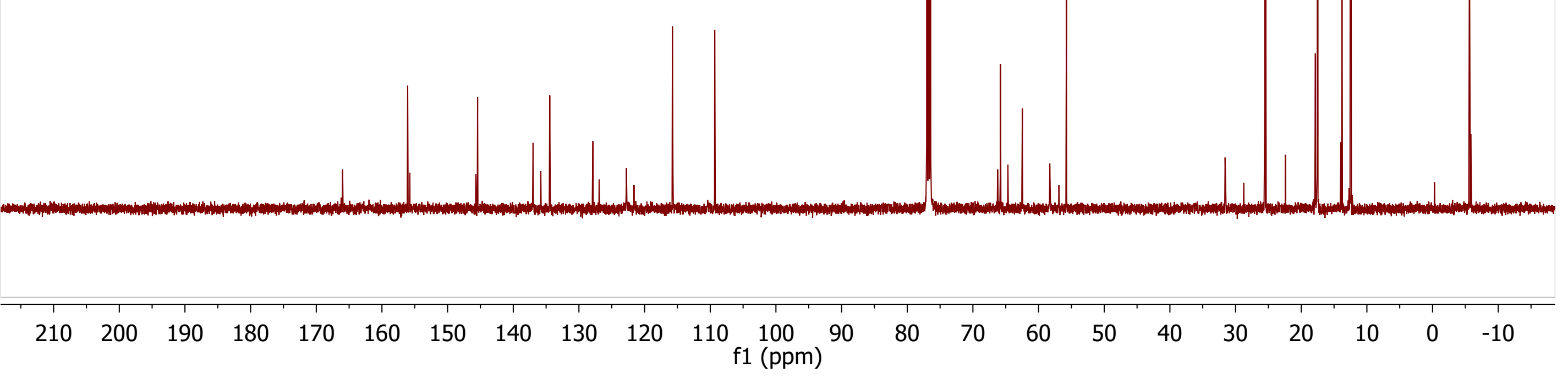


(E)-(2-(((tert-butyldimethylsilyl)oxy)methylene)-4-methylpyrrolidin-1-yl)

(5-methoxy-2-nitro-4-((triisopropylsilyl)oxy)phenyl)methanone (12)

\begin{tabular}{lllllllllllllllllllllllll}
\hline 210 & 200 & 190 & 180 & 170 & 160 & 150 & 140 & 130 & 120 & 110 & 100 & 90 & 80 & 70 & 60 & 50 & 40 & 30 & 20 & 10 & 0 & -10 &
\end{tabular}


(S)-(2-amino-5-methoxy-4-((triisopropylsilyl)oxy)phenyl)

(2-(((tert-butyldimethylsilyl)oxy)methyl)-4-methyl-2,5-dihydro-1H-pyrrol-1-yl)methanone

\begin{tabular}{|c|c|c|c|c|c|c|c|c|c|c|c|c|c|c|c|c|c|c|}
\hline 9.5 & 9.0 & 8.5 & 8.0 & 7.5 & 7.0 & 6.5 & 6.0 & 5.5 & $\begin{array}{l}5.0 \quad 4.5 \\
\text { 1 } 1(\mathrm{ppm})\end{array}$ & 4.0 & 3.5 & 3.0 & 2.5 & 2.0 & 1.5 & 1.0 & 0.5 & 0.0 \\
\hline
\end{tabular}


(S)-(2-amino-5-methoxy-4-((triisopropylsilyl)oxy)phenyl)

(2-(((tert-butyldimethylsilyl)oxy)methyl)-4-methyl-2,5-dihydro-1H-pyrrol-1-yl)methanone

$\begin{array}{lllllllllllllllllllllllllllllll}210 & 200 & 190 & 180 & 170 & 160 & 150 & 140 & 130 & 120 & 110 & 100 & 90 & 80 & 70 & 60 & 50 & 40 & 30 & 20 & 10 & 0 & -10\end{array}$




\section{Computational Estimates for the Thermodynamics of the Isomerisation Reactions}

The thermodynamics of double bond migration were studied with quantum chemistry.

All calculations were carried out with the Gaussian 16 (Rev C.01) software. All optimized structures were confirmed as being local minima from the absence of negative vibrational frequencies.

The work used computational approaches reported by Yu and Karton in their comprehensive computational study of the thermodynamics of conjugated to non-conjugate double bond isomerisation reactions. ${ }^{1}$ Three levels of theory were used for the study:

1. Accurate composite method CBS-QB3

2. B3LYP-D3BJ/cc-pVTZ

3. B3LYP-D3BJ/cc-pVTZ//M06-2X-D3/aug-cc-pVTZ

Calculations at the M06-2X-D3/aug-cc-pVTZ level have also ranked very well also in another comprehensive evaluation of computational benchmarks by Goerigk and Grimme. ${ }^{2}$

Double bond migration in compounds $6,8,11,12$ and 13 was studied using two model systems:

Model 1

A pared-down set of models, containing a minimal set of structural features $(\mathbf{6 a}, \mathbf{8 a}, \mathbf{1 1 a}, \mathbf{1 2 a}$ and 13a), were studied at levels 1,2 and 3 .<smiles>C=C1C[C@H](COC(C)=O)N(C(C)=O)C1</smiles>

$8 \mathbf{a}$<smiles>CC(=O)OC[C@@H]1C=C(C)CN1C(C)C</smiles>

$11 \mathrm{a}$<smiles>CC(=O)OC[C@@H]1CC(C)=CN1C(C)(F)F</smiles>

$6 a$<smiles>CC(=O)OCC1=C[C@@H](C)CN1C(C)C</smiles>

$13 a$<smiles>CC(=O)OC=C1C[C@H](C)CN1C(C)C</smiles>

$12 a$

\section{Model 2}

A realistic set of models, containing all major structural features of the original compounds, but with different protecting groups $(\mathbf{6 b}, \mathbf{8 b}, \mathbf{1 1 b}, \mathbf{1 2 b}$ and $\mathbf{1 3 b})$, were studied at levels 2 and 3 .<smiles>COc1cc(C(=O)N2CC(C)C[C@]2(COC(C)=O)OC(C)(C)C)c([N+](=O)[O-])cc1OC</smiles><smiles>COc1cc(C(=O)N2C=C(C)C[C@]2(COC(C)=O)OC(C)=O)c([N+](=O)[O-])cc1OC</smiles><smiles>COc1cc(C(=O)N2C[C@H](C)CC2COC(C)=O)c([N+](=O)[O-])cc1OC</smiles><smiles>COc1cc(C(=O)N2CC(C)=CC2(OC(C)=O)OC(C)=O)c([N+](=O)[O-])cc1OC</smiles><smiles>COc1cc(C(=O)N2C[C@H](C)C=C2OC(C)=O)c([N+](=O)[O-])cc1OC</smiles>

For all structures a preliminary conformation search was carried out the OPLS3 forcefield implemented in Schrodinger's Macromodel (Release 2019-2) and up to ten conformers of each structure taken into the quantum chemical studies. The relative enthalpies and free energies reported below refer to the most stable conformation found for each structure. 


\begin{tabular}{|c|c|c|c|c|c|c|c|}
\hline & \multicolumn{3}{|c|}{$\begin{array}{l}\text { Relative enthalpies }(\mathrm{kJ} / \mathrm{mol}) \text { of models } \\
\text { for structures }\end{array}$} & \multicolumn{3}{|c|}{$\begin{array}{l}\text { Relative free energies }(\mathrm{kJ} / \mathrm{mol}) \text { of } \\
\text { models for structures }\end{array}$} \\
\hline & & $\begin{array}{l}\hat{0} \\
0 \\
\tilde{1} \\
\tilde{0}\end{array}$ & 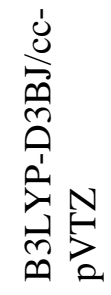 & 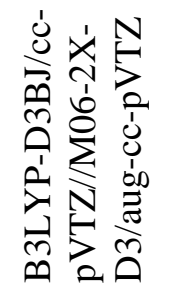 & $\begin{array}{l}\tilde{0} \\
0 \\
\tilde{1} \\
\tilde{0}\end{array}$ & 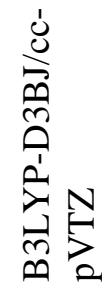 & 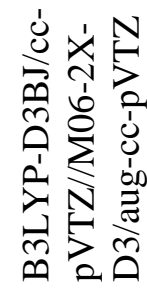 \\
\hline \multirow{5}{*}{$\begin{array}{l}\bar{\sigma} \\
\frac{\bar{d}}{0} \\
\dot{z}\end{array}$} & $8 \mathbf{a}$ & 0 & 0 & 0 & 0 & 0 & 0 \\
\hline & 11a & -17.3 & -16.5 & -15.6 & -20.5 & -19.7 & -18.8 \\
\hline & $6 a$ & -22.7 & -22.8 & -22.2 & -22.8 & -24.7 & -22.7 \\
\hline & $13 a$ & 1.2 & -0.4 & 1.5 & -3.0 & -6.3 & -4.2 \\
\hline & $12 a$ & 0.2 & -5.0 & -0.8 & -1.3 & -8.6 & -4.4 \\
\hline \multirow{5}{*}{$\begin{array}{l}N \\
\frac{0}{8} \\
\frac{0}{2}\end{array}$} & $8 b$ & 0 & 0 & 0 & 0 & 0 & 0 \\
\hline & $11 \mathrm{~b}$ & N/A & -16.7 & -16.5 & N/A & $\begin{array}{l}-19.2 \\
\end{array}$ & -17.2 \\
\hline & $6 \mathrm{~b}$ & N/A & -22.7 & -20.7 & N/A & -27.3 & -23.6 \\
\hline & $13 b$ & N/A & 8.4 & N/A & N/A & 9.9 & 1.5 \\
\hline & $12 \mathrm{~b}$ & N/A & -1.5 & 2.2 & N/A & -5.9 & -2.3 \\
\hline
\end{tabular}

Comparison of the numerical results between models and levels of theory reveals that the thermodynamics of double bond migration are consistent across models and levels of theory, lending credibility to the statement that $\mathbf{6}$ is the thermodynamic sink for the double bond migration. Values in the shaded boxes are reported in Scheme 2 of the paper.

\section{References}

1. Yu, L-J.; Karton, A. Assessment of theoretical procedures for a diverse set of isomerization reactions involving double-bond migration in conjugated dienes Chem. Phys. 2014, 441, 166177.

2. Goerigk, L.; Grimme, S. A thorough benchmark of density functional methods for general main group thermochemistry, kinetics, and noncovalent interactions Phys. Chem. Chem. Phys. 2011, 13, 6670-6688. 


\section{Chiral Analysis of 4-Methyl-2,3-dihydropyrrole (6)}

To demonstrate that the chiral centre was not racemised during the isomerisation, single enantiomers of (S)-(2-((tert-butyldimethylsilyl)oxy)methyl)-4-methylenepyrrolidin-1-yl)(5-methoxy-2-nitro-4((triisopropylsilyl)oxy)phenyl)methanone (6) and (R)-(2-((tert-butyldimethylsilyl)oxy)methyl)-4methylenepyrrolidin-1-yl)(5-methoxy-2-nitro-4-((triisopropylsilyl)oxy)phenyl)methanone were prepared using previously described methodology.

These were used to identify a chiral SFC method for separation of the enantiomers.

SFC Analysis:

Column: Lux Cellulose $23 \mu \mathrm{m}(150 \mathrm{~mm} \times 4.6 \mathrm{~mm})$

Mobile phase A: $\mathrm{CO}_{2}$

Mobile phase B: EtOH/MeOH/isopropylamine 50/50/0.1 v/v

Gradient:

\begin{tabular}{|l|l|l|}
\hline Time & $\% \mathrm{~A}$ & $\% \mathrm{~B}$ \\
\hline 0.00 & 95 & 5 \\
\hline 8.00 & 50 & 50 \\
\hline 10.00 & 50 & 50 \\
\hline
\end{tabular}

Flow rate: $2.5 \mathrm{~mL} / \mathrm{min}$

Detection: UV @ 254nm

Temperature: $35^{\circ} \mathrm{C}$

BPR: 150bar

Pseudoracemate

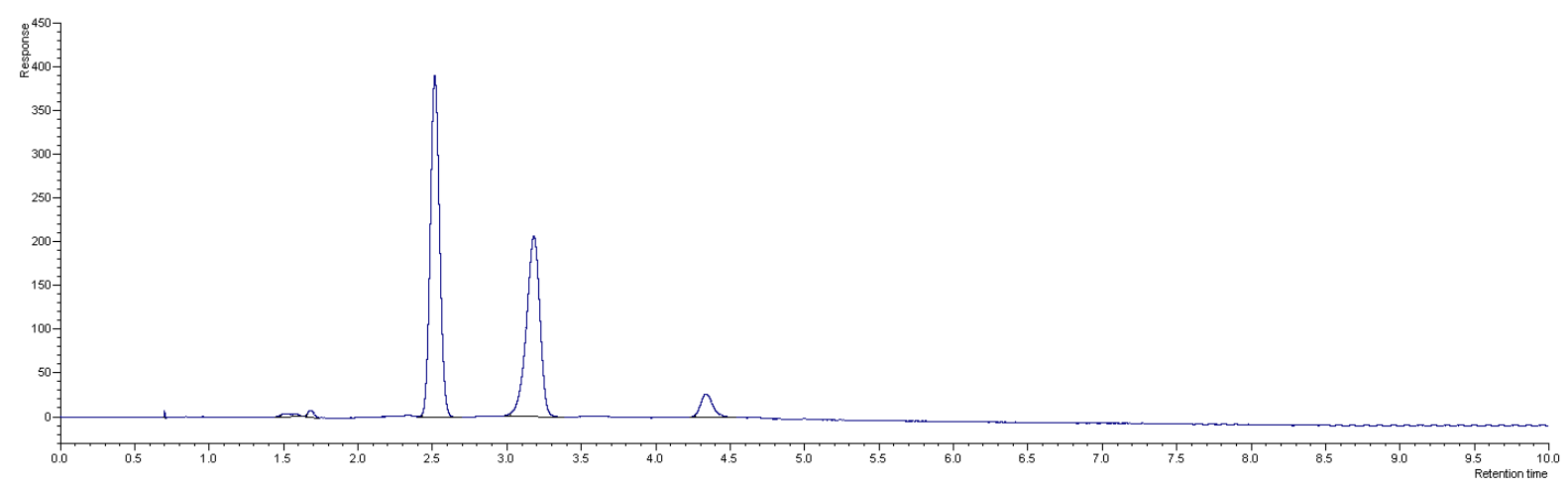


Sample of $(S)$-(2-amino-5-methoxy-4-((triisopropylsilyl)oxy)phenyl)(2-(( tert-

butyldimethylsilyl)oxy)methyl)-4-methyl-2,3-dihydro-1H-pyrrol-1-yl)methanone (6) prepared using isomerisation

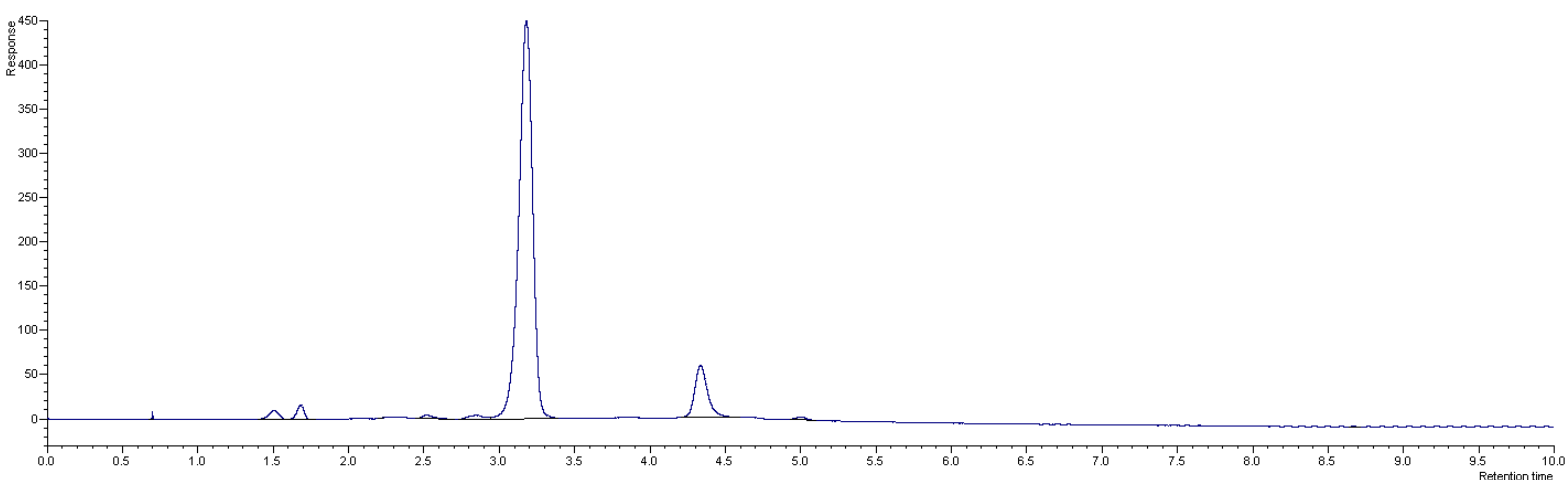

$(S)$-Isomer: $99.6 \%$

(R)-Isomer: $0.4 \%$ 
(S)-(2-amino-5-methoxy-4-((triisopropylsilyl)oxy)phenyl)

(2-(((tert-butyldimethylsilyl)oxy)methyl)-4-methyl-2,3-dihydro-1H-pyrrol-1-yl)methanone (6)

\begin{tabular}{|c|c|c|c|c|c|c|c|c|c|c|c|c|c|c|c|c|c|c|}
\hline 1 & 1 & 1 & $T$ & $T$ & $T$ & $T$ & $T$ & 1 & 1 & $T$ & $T$ & $T$ & $T$ & $T$ & $T$ & $T$ & $T$ & $T$ \\
\hline 9.5 & 9.0 & 8.5 & 8.0 & 7.5 & 7.0 & 6.5 & 6.0 & 5.5 & $\begin{array}{l}5.0 \quad 4.5 \\
\mathrm{f} 1(\mathrm{ppm})\end{array}$ & 4.0 & 3.5 & 3.0 & 2.5 & 2.0 & 1.5 & 1.0 & 0.5 & 0.0 \\
\hline
\end{tabular}


(S)-(2-amino-5-methoxy-4-((triisopropylsilyl)oxy)phenyl)

(2-((tert-butyldimethylsilyl)oxy)methyl)-4-methyl-2,3-dihydro-1H-pyrrol-1-yl)methanone (6)

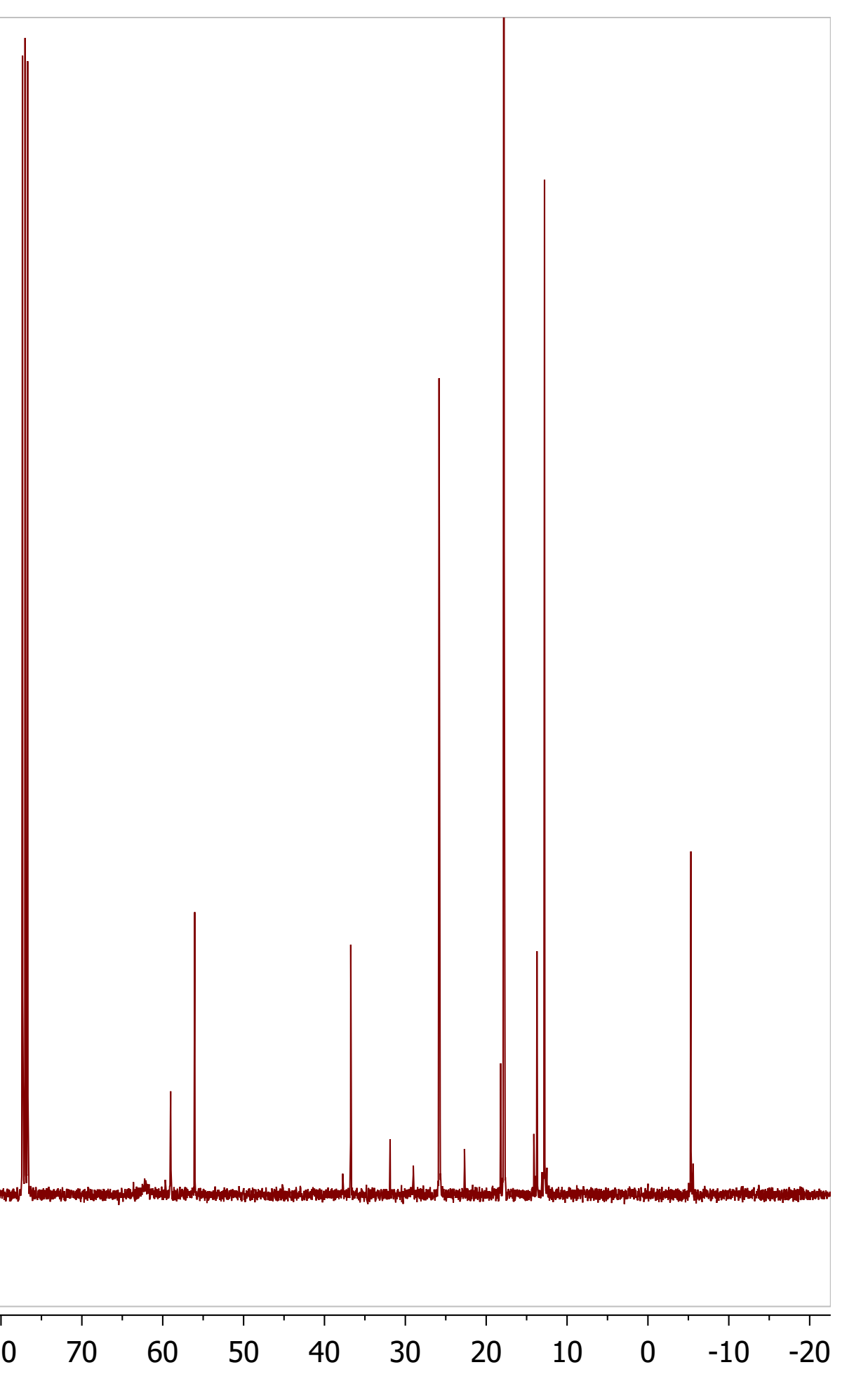

